\title{
Histone deacetylase 6-mediated selective autophagy regulates COPD-associated cilia dysfunction
}

Hilaire C. Lam, ${ }^{1,2,3}$ Suzanne M. Cloonan, ${ }^{1}$ Abhiram R. Bhashyam, ${ }^{1}$ Jeffery A. Haspel,1,4 Anju Singh, ${ }^{5}$ J. Fah Sathirapongsasuti, 6,7 Morgan Cervo, ${ }^{8}$ Hongwei Yao, ${ }^{9}$ Anna L. Chung, ${ }^{1}$ Kenji Mizumura, ${ }^{1}$ Chang Hyeok An, ${ }^{1}$ Bin Shan, ${ }^{10}$ Jonathan M. Franks, ${ }^{11}$ Kathleen J. Haley, ${ }^{1}$

Caroline A. Owen, ${ }^{1}$ Yohannes Tesfaigzi, ${ }^{3}$ George R. Washko, ${ }^{1}$ John Quackenbush,6,7

Edwin K. Silverman, ${ }^{12}$ Irfan Rahman, ${ }^{9}$ Hong Pyo Kim, ${ }^{1,13}$ Ashfaq Mahmood, 8 Shyam S. Biswal, ${ }^{5}$ Stefan W. Ryter, ${ }^{1,3}$ and Augustine M.K. Choi ${ }^{1}$

1Division of Pulmonary and Critical Care Medicine, Brigham and Women's Hospital and Harvard Medical School, Boston, Massachusetts, USA. 2Department of Pathology, University of Pittsburgh School of Medicine, Pittsburgh, Pennsylvania, USA. ${ }^{3}$ Lovelace Respiratory Research Institute,

Albuquerque, New Mexico, USA. 'Pulmonary and Critical Care Medicine Section, VA Boston Healthcare System, Boston, Massachusetts, USA.

${ }^{5}$ Department of Environmental Health Sciences, Johns Hopkins University, Bloomberg School of Public Health, Baltimore, Maryland, USA.

${ }^{6}$ Department of Biostatistics, Harvard School of Public Health, Boston, Massachusetts, USA. 7Department of Biostatistics and Computational Biology,

Dana-Farber Cancer Institute, Boston, Massachusetts, USA. ${ }^{8}$ Department of Radiology, Brigham and Women's Hospital and Harvard Medical School,

Boston, Massachusetts, USA. 9University of Rochester, Environmental Medicine, Rochester, New York, USA. ${ }^{10}$ Department of Medicine,

Tulane University School of Medicine, New Orleans, Louisiana, USA. ${ }^{11}$ Center for Biological Imaging, University of Pittsburgh, Pittsburgh, Pennsylvania, USA.

${ }^{12}$ Channing Laboratory, Brigham and Women's Hospital, Boston, Massachusetts, USA. ${ }^{13}$ School of Pharmacy, Ajou University, Suwon, Republic of Korea.

Chronic obstructive pulmonary disease (COPD) involves aberrant airway inflammatory responses to cigarette smoke (CS) that are associated with epithelial cell dysfunction, cilia shortening, and mucociliary clearance disruption. Exposure to CS reduced cilia length and induced autophagy in vivo and in differentiated mouse tracheal epithelial cells (MTECs). Autophagy-impaired (Becn1 ${ }^{+/-}$or Map $\left.1 l c 3 \mathrm{~B}^{-/-}\right)$mice and MTECs resisted CS-induced cilia shortening. Furthermore, CS increased the autophagic turnover of ciliary proteins, indicating that autophagy may regulate cilia homeostasis. We identified cytosolic deacetylase HDAC6 as a critical regulator of autophagy-mediated cilia shortening during CS exposure. Mice bearing an X chromosome deletion of Hdac6 (Hdac6-/Y) and MTECs from these mice had reduced autophagy and were protected from CS-induced

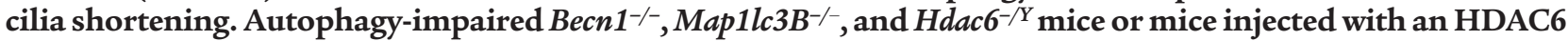
inhibitor were protected from CS-induced mucociliary clearance (MCC) disruption. MCC was preserved in mice given the chemical chaperone 4-phenylbutyric acid, but was disrupted in mice lacking the transcription factor NRF2, suggesting that oxidative stress and altered proteostasis contribute to the disruption of MCC. Analysis of human COPD specimens revealed epigenetic deregulation of HDAC6 by hypomethylation and increased protein expression in the airways. We conclude that an autophagy-dependent pathway regulates cilia length during CS exposure and has potential as a therapeutic target for COPD.

\section{Introduction}

Chronic obstructive pulmonary disease (COPD) contributes substantially to the global burden of disease as the fourth leading cause of morbidity and mortality worldwide (1). The pathogenesis of COPD remains poorly understood, but involves aberrant inflammatory and cellular responses of the lung caused by chronic cigarette smoke (CS) exposure (2). The complex pathology of this disease results in two major clinical phenotypes: bronchitis associated with airway inflammation and mucus obstruction and emphysema characterized by loss of alveolar surface area for gas exchange (3).

Epithelial cells lining the airways and alveoli represent a primary target of inhaled CS. The mechanisms underlying CS-induced epithelial cell injury and dysfunction remain unclear, but may include a protease/antiprotease imbalance, inflammation, oxidative stress,

Authorship note: Hilaire C. Lam and Suzanne M. Cloonan contributed equally to this work.

Conflict of interest: The authors have declared that no conflict of interest exists. Citation for this article: J Clin Invest. 2013;123(12):5212-5230. doi:10.1172/JCI69636. and programmed cell death (2-4). Recent studies have suggested additional pathway mechanisms involving altered protein homeostasis (proteostasis) $(5,6)$, including ER stress, inhibition of the ubiquitin-proteasome system (7-9), and autophagy (10-13), all of which potentially contribute to the pathogenesis of chronic lung diseases and emphysema (5).

Autophagy refers to a dynamic process by which cytoplasmic organelles and proteins are sequestered into autophagosomes that subsequently fuse with lysosomes, leading to the degradation of cargo by lysosomal hydrolases $(14,15)$. At least thirty autophagy-related (Atg) proteins regulate autophagy in eukaryotes (16). Among these, beclin 1 (the mammalian homolog of yeast Atg6) represents a major upstream autophagic regulator (17). Beclin 1 associates with a macromolecular complex that includes the class III phosphatidylinositol-3 kinase (Vps34), which produces phosphatidylinositol-3-phosphate, a second messenger that regulates autophagosomal nucleation (18). Microtubule-associated protein light chain (LC3, a homolog of yeast Atg8), a ubiquitin-like protein, assimilates into maturing 
autophagosomes (19). Conversion of LC3-I (cytosolic form) to its phosphatidylethanolamine-conjugated form (LC3B-II) represents a key step in autophagosome formation (19).

While the ubiquitin-proteasome system functions as the primary mechanism for cellular protein turnover (20), ubiquitinated or misfolded proteins that are not degraded by the proteasome form insoluble aggregates that are degraded by autophagy (21). The specialized degradation of denatured proteins by autophagy involves a selective pathway (aggrephagy) by which ubiquitinated protein aggregates are organized into inclusion bodies (aggresomes) and then targeted to autophagosomes by specific adaptor proteins (e.g., p62/SQSTM1) (22-24).

In the lungs, the mucociliary escalator acts as a primary innate defense mechanism, in which motile ciliated epithelial cells eliminate particles and pathogens trapped in mucus from the airways. Disruption of airway epithelial cell function upon CS exposure results in impaired mucociliary clearance (MCC) (25-27). In patients with COPD, impaired airway clearance may promote susceptibility to respiratory infections (2). Disruption of MCC in response to CS exposure is attributed to a reduction in epithelial cell cilia length and airway epithelial cell death (4, 25-27), followed by reepithelialization dominated by goblet cells, resulting in excess mucus production (28). The mechanisms by which CS-induced epithelial cell dysfunction leads to cilia shortening and altered airway function in vivo remain unclear. Currently, few advances have been made to alleviate MCC disruption and bronchitis associated with the pathogenesis of COPD, with most therapeutic options focusing on inhaled bronchodilators and corticosteroids (29).

Ciliated cells of the respiratory tract have large numbers of motile cilia, each nucleated by a basal body, from which extends the ciliary axoneme (30). Motile cilia are dynamic organelles that depend on ATP-driven motor proteins for motility and on intraflagellar transport for structural maintenance (31). Recent observations in single-celled organisms suggest that cilia resorption or shortening requires the ubiquitination of ciliary proteins followed by cytoplasmic translocation for degradation (32). Primary cilia disassembly and shortening have been previously attributed to several signaling molecules, including the human enhancer of filamentation protein 1 (HEF1), aurora A (AurA), histone deacetylase 6 (HDAC6) and glycogen synthase kinase $\beta$ (GSK3 $\beta)(33,34)$. Little is known about the processes that regulate motile ciliary length in human airways.

Recent studies suggest that cytosolic and/or histone deacetylases (HDACs) represent novel therapeutic targets for modulation of the autophagic pathway (35). Among these, the cytosolic deacetylase HDAC6, which contains ubiquitin-binding and dynein-interacting domains, has emerged as a pleiotropic regulator of cellular function. HDAC6 controls diverse cellular processes through deacetylating and destabilizing microtubules (33), facilitating the retrograde transport of ubiquitinated proteins into aggresomes $(36,37)$ and enhancing autophagosomelysosome fusion (38). The roles of HDAC6 in motile cilia of the airways, in cellular responses to CS exposure, and in COPD pathogenesis have not been previously studied.

We have previously demonstrated increased autophagic protein expression and autophagosome accumulation in cultured epithelial cells and in the lungs of COPD patients (10). In the current study, we examined the mechanism(s) by which autophagy regulates airway function and ciliated epithelial cell length during CS exposure. We also examined the mechanisms by which ubiq- uitination and/or autophagy can promote the turnover of mammalian ciliary proteins in experimental and human COPD. We report here that HDAC6 and other regulators of the autophagic pathway adversely impact cilia length, phenotypic responses, and functional airway outcomes during CS exposure in a process we term "ciliophagy." Our findings suggest new therapeutic targets for improving airway function during chronic lung diseases such as COPD through the maintenance of epithelial cell proteostasis and modulation of the autophagic pathway.

\section{Results}

CS causes cilia shortening in primary airway epithelial cell cultures associated with a proautophagic phenotype. We sought to understand the mechanisms by which CS exposure disrupts the function of ciliated epithelial cells of the respiratory tract and their impact on airway function. As cultured epithelial cell lines or primary cell lines cannot form motile cilia, we developed a physiologically relevant model of highly differentiated mouse tracheal epithelial cells (MTECs) grown at the air-liquid interface (ALI). To simulate the effects of CS on cilia homeostasis in the lung, we used a novel microenvironment total particulate matter-controlled (TPM-controlled) chamber in which the apical surface of MTEC cultures was exposed to mainstream CS (39). Using this model, we observed that CS exposure exerted dose-dependent effects on the morphology and viability of MTECs.

Immunofluorescence imaging using acetylated $\alpha$-tubulin demonstrated dose-dependent changes in ciliated cell morphology in MTECs after a 24-hour exposure to CS $\left(50-100 \mathrm{mg} / \mathrm{m}^{3}\right)$. Cultures exposed to $50 \mathrm{mg} / \mathrm{m}^{3}$ CS displayed cilia shortening, whereas substantial ciliated cell loss occurred only at a dose of $100 \mathrm{mg} / \mathrm{m}^{3} \mathrm{CS}$ (Figure 1A). Immunofluorescence analysis did not reveal evidence of detached cilia in apical washes (H.C. Lam, unpublished observations).

Cultures that were treated with CS at a TPM dose of $50 \mathrm{mg} / \mathrm{m}^{3}$ recovered epithelial integrity and retained viability 24 hours after treatment. However, cultures exposed to CS at $100 \mathrm{mg} / \mathrm{m}^{3}$ lost integrity and viability, as determined by the decline in transepithelial electrical resistance (TER) (Supplemental Figure 1A; supplemental material available online with this article; doi:10.1172/JCI69636DS1), increased lactate dehydrogenase (LDH) release into the media (Supplemental Figure 1B), and loss of cellular ATP (Supplemental Figure 1C). Furthermore, a dose of $50 \mathrm{mg} / \mathrm{m}^{3} \mathrm{CS}$ did not induce apoptosis, whereas $100 \mathrm{mg} / \mathrm{m}^{3}$ increased the number of apoptotic cells as determined by annexin $\mathrm{V}$-positive and propidium iodide-negative staining (Supplemental Figure 1D) or scanning electron microscopy (SEM) of cell morphology (Supplemental Figure 1E).

To further understand the effect of CS on cilia morphology, we analyzed cilia length in MTECs subjected to CS by SEM imaging and quantification (Figure 1, B-C). CS exposure at doses of $50 \mathrm{mg} / \mathrm{m}^{3}$ (Figure 1, B-C) and $100 \mathrm{mg} / \mathrm{m}^{3}$ (data not shown) dramatically increased the population of cells with shortened cilia. CS caused a dose-dependent reduction in mean cilia length (Figure 1C). To accurately assess the extent of cilia shortening in MTEC cultures, we used an empiric threshold of $0.6 \mathrm{AU}$ to filter the cilia length frequency histogram (Figure 1B) into "short" and "long" cilia length morphology (40) (see detailed description in Methods). Using this analysis, we show that CS dose-dependently reduced the number of epithelial cells scoring for long cilia morphology. Taken together, these data indicate that cilia shortening, rather than ciliated 

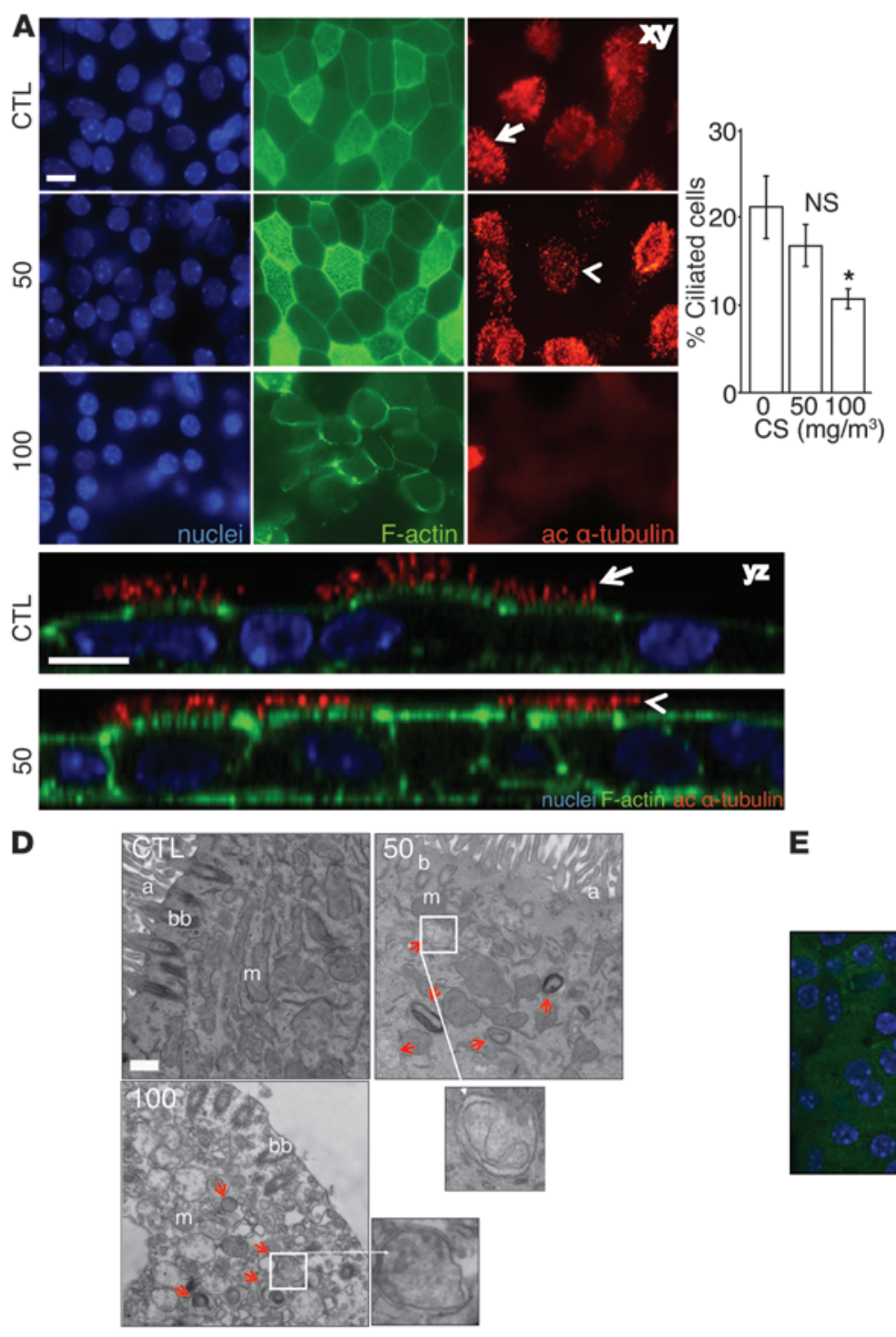

E
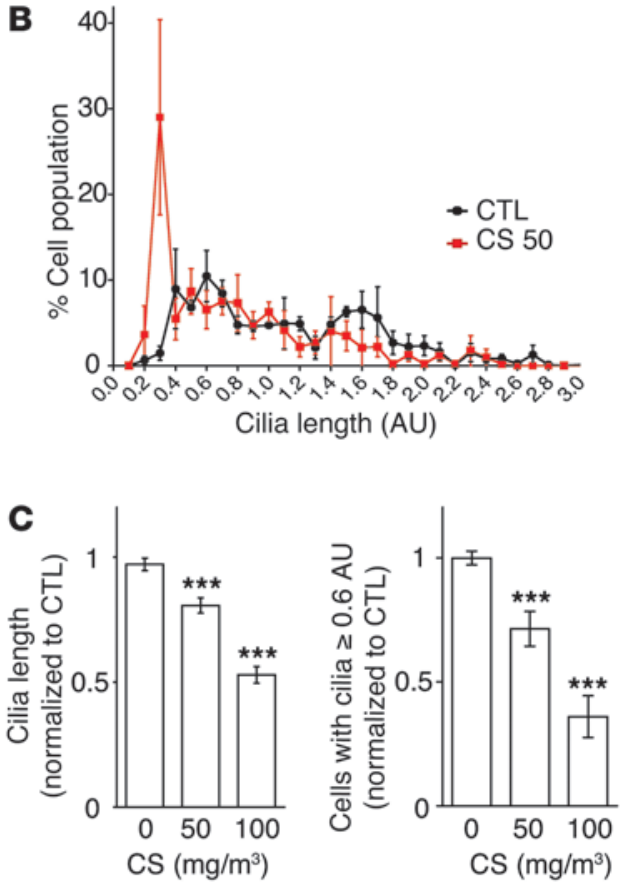

CTL

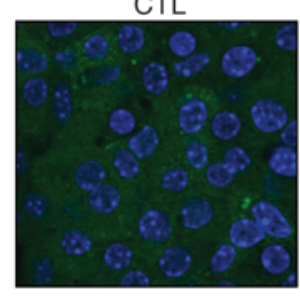

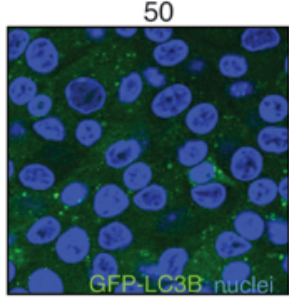

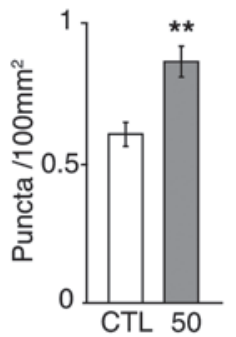

\section{Figure 1}

Cigarette smoke causes cilia loss and shortening and increased autophagy in primary cultured epithelial cells. (A) Cells exposed to 50 or $100 \mathrm{mg} / \mathrm{m}^{3}$ were harvested 24 hours after exposure and analyzed for the cilia marker acetylated $\alpha$-tubulin, F-actin, and Hoechst staining by confocal $x y$ - or $z$-stack analysis. Scale bar: $10 \mu \mathrm{m}$. Arrows indicate intact cilia; arrowheads indicate shortened cilia. Dara are representative of three independent experiments. Quantification of ciliated cells (right) was performed based on the morphological appearance of acetylated $\alpha$-tubulin-positive cells normalized to total nuclei. (B) Distribution of cilia lengths by SEM in MTECs 24 hours after exposure to $50 \mathrm{mg} / \mathrm{m}^{3} \mathrm{CS}$. Distributions were constructed by creating histograms from $0.2 \mathrm{AU}$ bins, equally weighting each sample. (C) Average cilia length and change in the number of cells with cilia lengths of $0.6 \mathrm{AU}$ or more in MTECs exposed to 50 or $100 \mathrm{mg} / \mathrm{m}^{3} \mathrm{CS}$. Cilia lengths were calculated from $10 \mathrm{SEM}$ images/MTEC culture $(n=3)$. The number of MTECs with cilia lengths of $0.6 \mathrm{AU}$ or more were calculated from 5 SEM images per sample ( $n=4$ cultures/group) normalized to controls. (D) Ciliated MTECs were analyzed for ultrastructural changes 24 hours after exposure to CS at the indicated doses. Red arrows indicate autophagosomes/autophagolysosomes. a, axoneme; bb, basal body; $\mathrm{m}$, mitochondria. Scale bar: $500 \mathrm{~nm}$. Image is representative of 45 images ( $n=1$ MTEC culture). (E) Autophagosome-associated GFP-LC3 puncta were imaged and quantified (right) by confocal microscopy in control- and CS-treated ( $\left.50 \mathrm{mg} / \mathrm{m}^{3}\right) \mathrm{MTECs}(5 \mathrm{images} / \mathrm{culture}$ from 3 MTEC cultures). All data are the mean \pm SEM. ${ }^{*} P<0.05$, ${ }^{* \star} P<0.01$, and ${ }^{* * *} P<0.001$ by one-way ANOVA followed by a Bonferroni's post test.

epithelial cell death, is the major phenotype of epithelial cells exposed to acute $50 \mathrm{mg} / \mathrm{m}^{3} \mathrm{CS}$ exposure and that loss of epithelial cell integrity occurs at the higher dose of $100 \mathrm{mg} / \mathrm{m}^{3} \mathrm{CS}$ exposure.

Next, we performed ultrastructural analysis of MTECs subjected to CS exposure using transmission electron microscopy (TEM). CS exposure $\left(50 \mathrm{mg} / \mathrm{m}^{3}\right)$ increased autophagosome formation in ciliated cells (Figure 1D). Consistently, CS exposure at this dose caused increases in GFP-LC3 puncta formation in MTECs isolated from GFP-LC3B mice, indicative of autophagosome formation (Figure 1E). Consistent with our toxicological observations (Supplemental Figure 1), TEM analysis of MTECs exposed to CS at $100 \mathrm{mg} / \mathrm{m}^{3}$ demonstrated cellular degeneration with excess autophagic vacuole formation and significant ciliary degeneration (Figure 1D). 
A
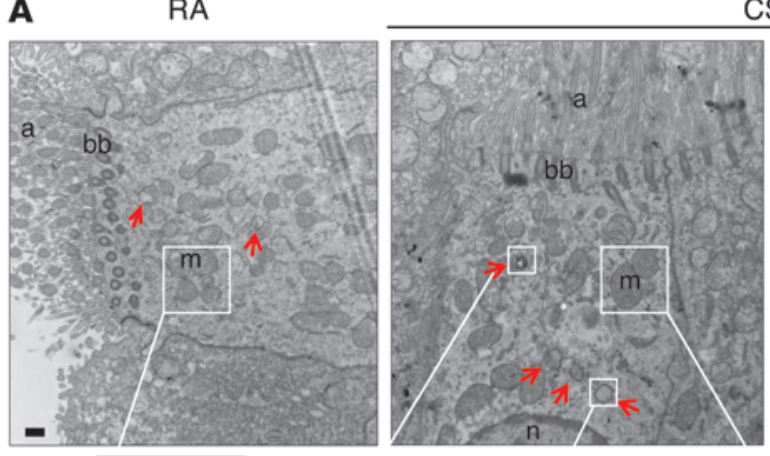

CS
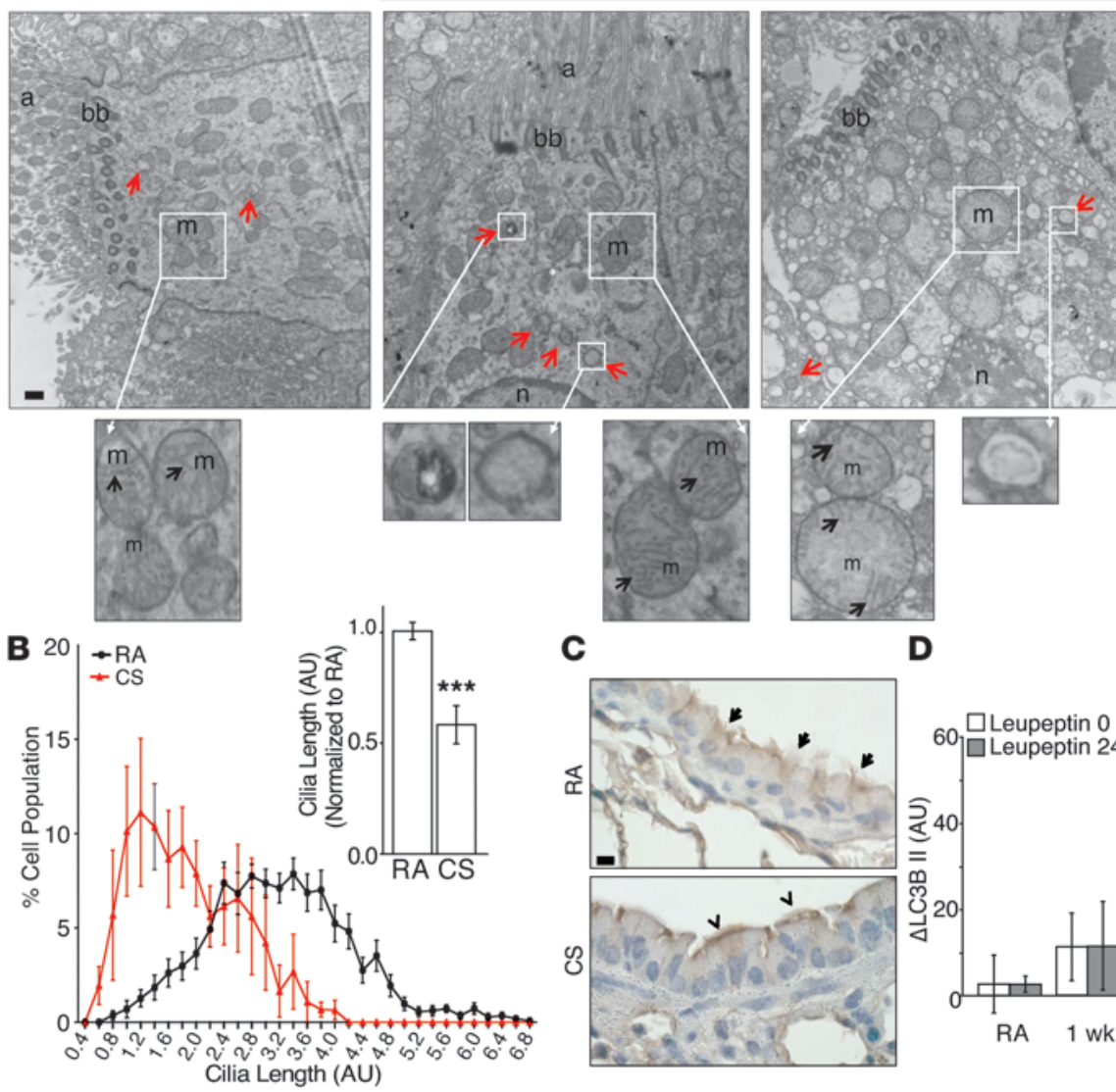

C
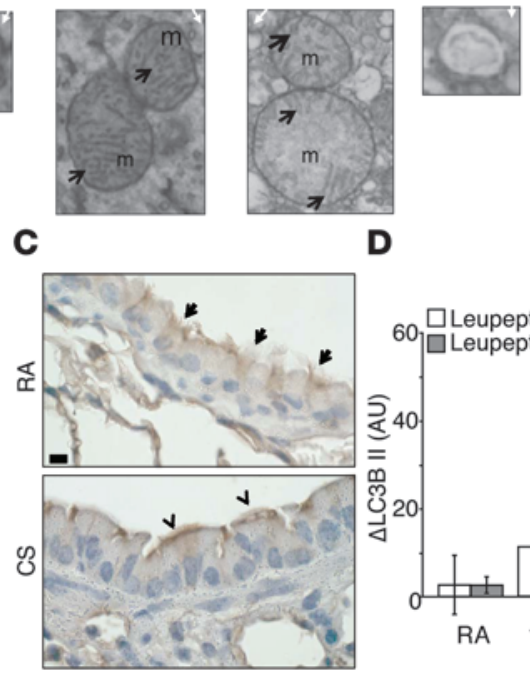

D

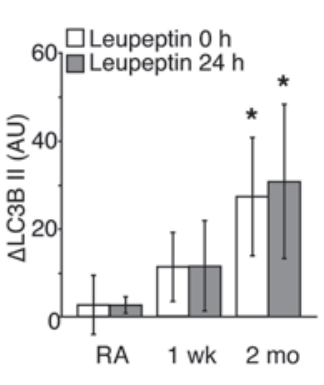

\section{Figure 2}

CS causes cilia shortening and increased autophagy in vivo. (A) Ciliated mouse tracheal cells were analyzed by TEM after a 1-week exposure to CS or RA in vivo. Image is representative of 15 EM fields taken from 2 to 3 mice. Scale bar: $500 \mathrm{~nm}$. Red arrows indicate autophagosomes. $n$, nuclei; $\mathrm{m}$, mitochondria; black arrows indicate mitochondrial cristae. (B) Distribution of cilia lengths and the average cilia length of H\&E-stained airways exposed to RA or CS for 2 months. Distributions were constructed by creating histograms from $0.4 \mathrm{AU}$ bins, equally weighting each sample. The average cilia length (right) was calculated by measuring a minimum of 3 cilia in every multiciliated cell and normalizing to CTL ( $n=3-4 /$ group, $10 \mathrm{H \& E}$-stained images/sample). (C) Ciliated cells of mouse respiratory epithelium following 3 months of RA or CS exposure were immunohistochemically labeled by IFT88. Scale bar: $10 \mu \mathrm{m}$. Arrows indicate intact airway cilia; arrowheads indicate shortened airway cilia. Image is representative of 3 to 4 determinations. (D) Autophagic flux, as measured by LC3B turnover in the lungs of mice exposed to RA $(n=3)$ or CS $(n=3)$ for 1 week or 2 months, was determined immediately after the final exposure to CS ( $t=0$ hours) or 24 hours later ( $t=24$ hours) by injecting with the autophagic inhibitor leupeptin (40 mg/kg), as described in Methods. All data are the mean \pm SEM. ${ }^{*} P<0.05$ and ${ }^{* * *} P<0.001$ by an unpaired Student's $t$ test (B), or by one-way ANOVA followed by a Bonferroni's post test (D).
To determine whether increased autophagosome formation correlated with autophagic activity in MTECs treated with $50 \mathrm{mg} / \mathrm{m}^{3} \mathrm{CS}$, we implemented in vitro autophagic flux assays. These assays were designed to provide an estimate of intrinsic autophagic activity present in the MTECs following CS exposure by assessing the steady-state levels of the autophagic substrates LC3B and p 62 in the absence and presence of chloroquine (CQ), an inhibitor of lysosomal acidification (41-43). Analysis of autophagic flux in MTECs treated with CS $\left(50 \mathrm{mg} / \mathrm{m}^{3}\right)$ revealed that CS caused an immediate increase in the lysosomal degradation of LC3B and p62, suggestive of increased autophagic activity (Supplemental Figure 2, A-C).

Similar to observations in MTECs, autophagosome accumulation in conjunction with cilia shortening was observed in differentiated human epithelial cell cultures exposed to aqueous cigarette smoke extract (CSE) by TEM and SEM of the apical cell surface (Supplemental Figure 3, A and B). Taken together, these results indicate that CS can cause cilia shortening and concomitant increases in markers of autophagy, whereas elevated doses of CS (e.g., $100 \mathrm{mg} / \mathrm{m}^{3}$ ) cause epithelial cell death.
CS causes cilia shortening in the airways in vivo that is associated with increased autophagy. To study the effects of CS on airway morphology, we used an in vivo model of CS exposure. C57Bl/ 6 mice were exposed in modular chambers to room air (RA) or to CS for 2 hours per day $\left(150 \mathrm{mg} / \mathrm{m}^{3}\right), 5$ days per week for up to 6 months. Using this model, we assessed the effects of CS on the ultrastructure of ciliated cells of the airway using TEM. Similar to our in vitro observations, viable airway cells exposed to CS for 1 week displayed increased numbers of autophagosomes, whereas some individual cells displayed morphological features of cell death, were devoid of ciliary axonemes, and contained numerous degradative autophagic vacuoles (Figure 2A).

Quantification of cilia from H\&E-stained lung sections containing large airways (diameter $>200 \mu \mathrm{m}$ ) revealed that 2 months of CS exposure dramatically increased a population of cells with shortened cilia and caused a reduction in mean cilia length (Figure 2B). Immunohistochemical staining of airway epithelia using the ciliary protein IFT88 revealed marked cilia shortening and loss after 3 months of CS exposure in vivo (Figure $2 \mathrm{C}$ ). 


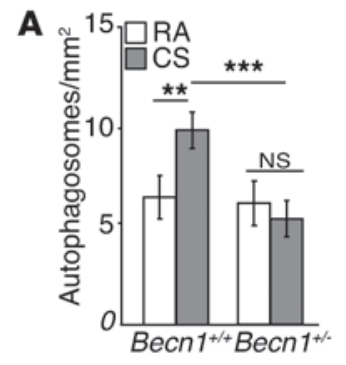

B
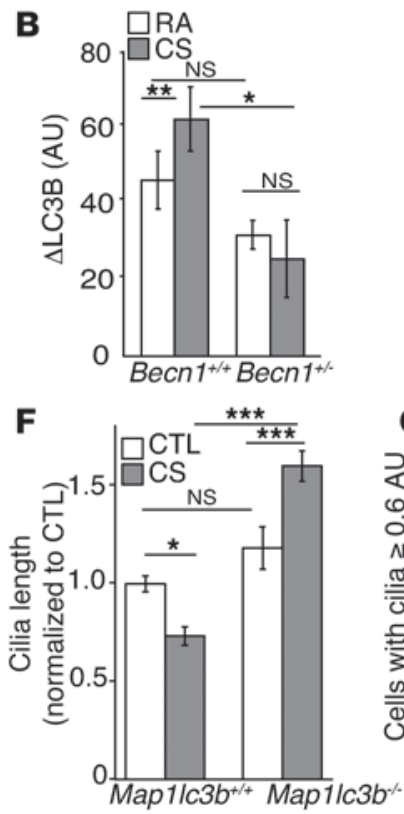

C
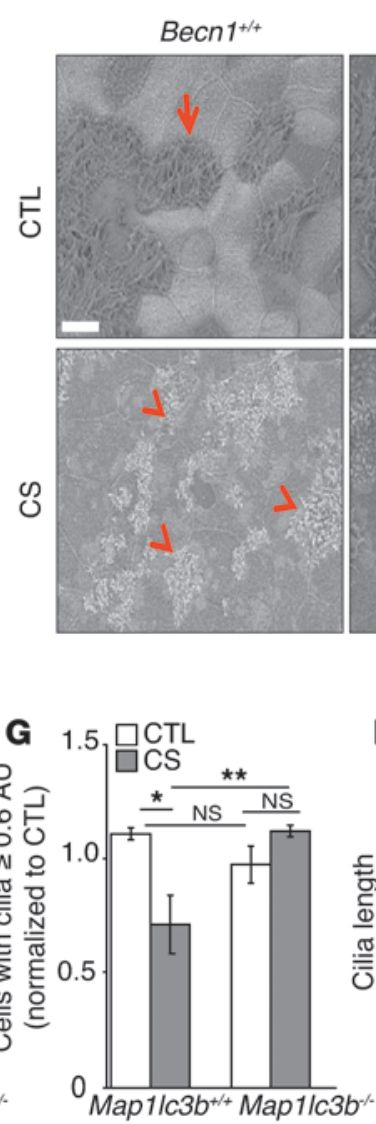

Becn1+-
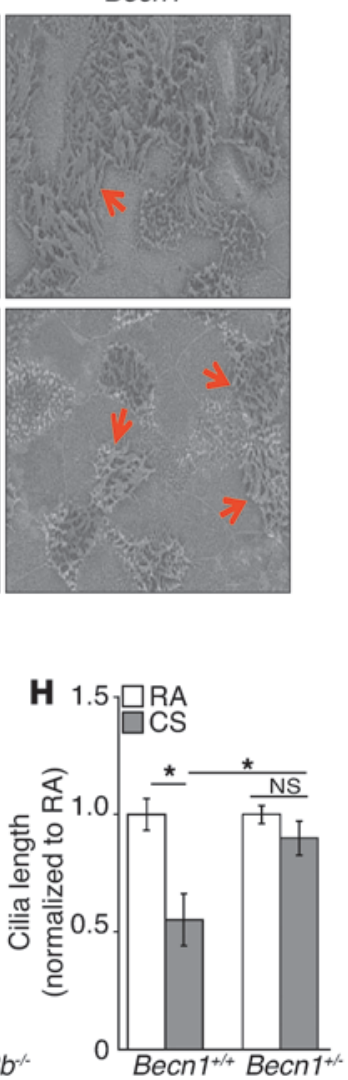
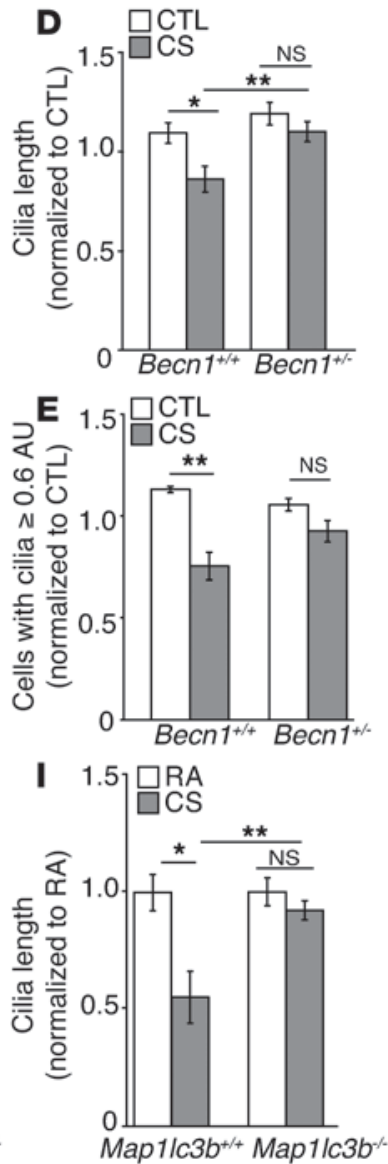

\section{Figure 3}

Autophagy impairment prevents cilia shortening in response to CS. (A) Autophagosomes per area were quantified from TEM images (10-15 images/mouse) of ciliated airway epithelial cells in Becn1+/+ and Becn1+/- mice exposed to RA or CS for 6 months ( $n=2-4 / g r o u p)$. (B) Autophagic flux was assessed by LC3B turnover in Becn1+/+ and Becn1+/- mice exposed to CS or RA for 6 months ( $n=3-4$ mice/group). (C) Representative SEM in Becn1+/+ and Becn1+/- MTECs 24 hours after treatment with CS (50 mg/m³). Red arrows indicate intact cilia; red arrowheads indicate shortened cilia. Scale bar: $500 \mathrm{~nm}$. (D) Change in cilia length and (E) cells with cilia lengths of $0.6 \mathrm{AU}$ or more in Becn1+/+ and Becn1+- MTECs 24 hours after treatment with CS $\left(50 \mathrm{mg} / \mathrm{m}^{3}\right)$. (F) Change in cilia length and $(\mathbf{G})$ cells with cilia lengths of 0.6 AU or more in Map1/c3 $b^{+/+}$and Map 1/c $3 b^{-/-}$MTECs 24 hours after treatment with CS $\left(50 \mathrm{mg} / \mathrm{m}^{3}\right)$. MTEC cilia lengths were calculated from 10 SEM images per experiment $(n=3)$. The number of MTECs with cilia lengths of $0.6 \mathrm{AU}$ or more were calculated from 5 SEM images per sample

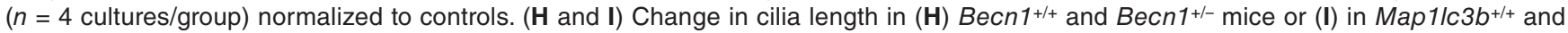
Map 1/c $3 \mathrm{~b}^{-/-}$mice exposed to CS for 2 months ( $n=4$ mice/group; $10 \mathrm{H \& E}$-stained images/mouse). The average cilia length per image was calculated by measuring $>3$ cilia for every ciliated cell and normalizing to controls. All data are the mean \pm SEM. ${ }^{\star} P<0.05$, ${ }^{\star \star} P<0.01$, and ${ }^{* * *} P<0.001$ by one- or two-way ANOVA and Bonferroni's post tests.

To determine whether increased autophagosome formation correlated with autophagic activity in the lungs of CS-treated mice, we implemented in vivo autophagic flux assays developed in our laboratory (44). These assays were designed to provide an estimate of intrinsic autophagic activity present in the tissue immediately following and the first 24 hours after smoking cessation. Mice subjected to RA or CS for 1 week or 2 months received a single i.p. injection of leupeptin, which inhibits lysosomal degradation of proteins, at 0 hours or 24 hours following smoking cessation. Subsequently, mice were sacrificed 2 hours after injection, and the lungs were harvested for protein. Autophagic flux was calculated as the difference in steady-state levels of LC3B in the absence or presence of leupeptin. Analysis of LC3B steady-state levels in a lysosome-enriched fraction of lung homogenates revealed the time-dependent increase in leupeptin-sensitive LC3B degradation in vivo, which persisted 24 hours after CS exposure (Figure 2D and Supplemental Figure 2D). These results support the conclusion that CS caused a cumulative increase in autophagic flux in lung tissue. Taken together, these results indicate that CS causes ciliated epithelial cell shortening and cilia dysfunction in vivo in association with increased autophagy.

Becn $1^{+/-}$epithelial cells are resistant to CS-induced cilia shortening. Given that increased indicators of autophagy correlated with cilia shortening in response to CS, we hypothesized that modulation of the autophagic pathway could affect cilia length. To study the relationship between autophagy and cilia length, we used a mouse model of autophagy deficiency with beclin 1 heterozygous knockout mice $\left(B e c n 1^{+-}\right)$(45). Becn1 ${ }^{+/-}$mice were confirmed to 
A

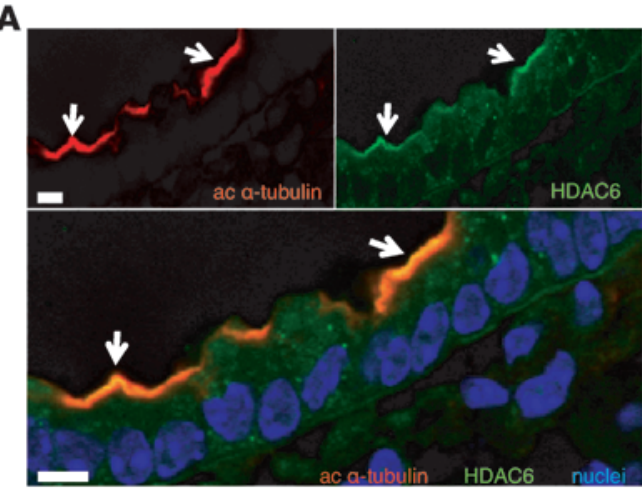

D
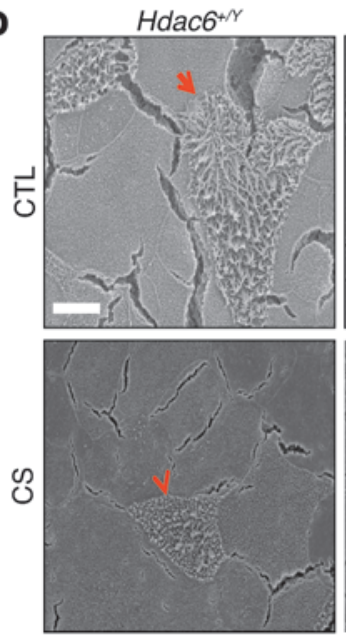
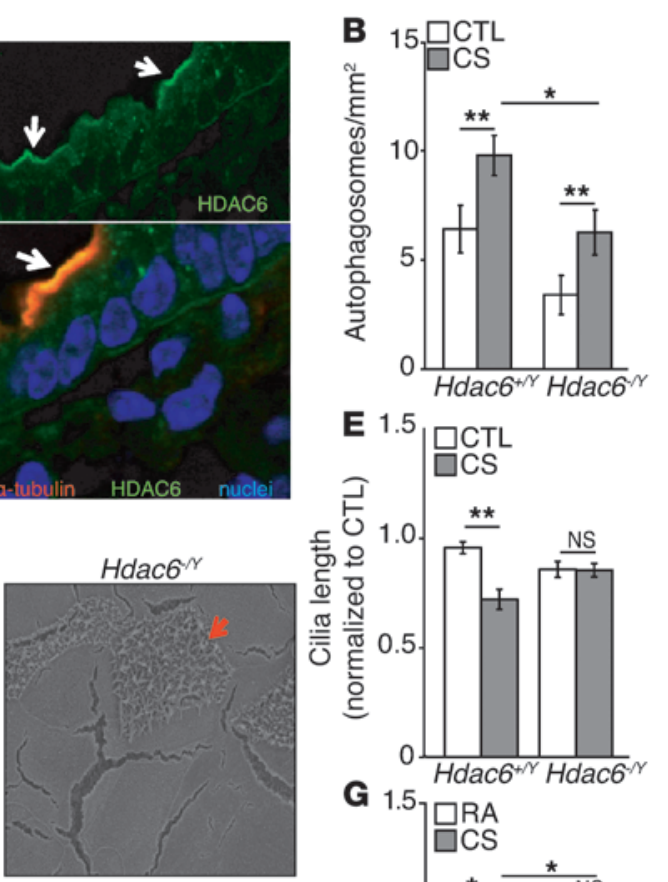

G 1
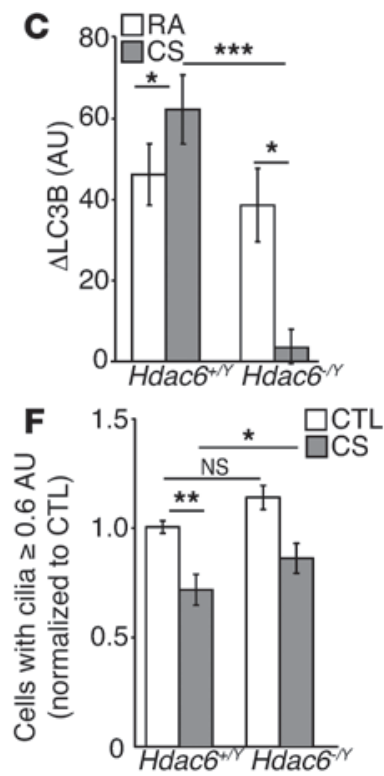

\section{Figure 4}

HDAC6 deficiency prevents cilia shortening in response to CS. (A) HDAC6 colocalized with acetylated $\alpha$-tubulin (arrows) in mouse airway cryosections. Image is representative of three to four determinations. Scale bar: $10 \mu \mathrm{m}$. (B) Autophagosomes were quantified in tracheal epithelial cells of $\mathrm{Hdac}^{+/ Y}$ ( $n=4 /$ group) and Hdac6 $6^{-/ Y}$ mice ( $n=2 /$ group) exposed to RA or CS for 6 months. (C) Autophagic flux was assessed by LC3B turnover in the lungs of $\mathrm{Hdac}^{+/ Y}$ and $\mathrm{Hdac}^{-/ Y}$ mice $\left(n=3-4 /\right.$ group) following 6 months of CS exposure. (D) $H d a c 6^{+/ Y}$ and $H d a c 6^{-/ Y}$ MTEC control cultures or MTECs exposed to $50 \mathrm{mg} / \mathrm{m}^{3} \mathrm{CS}$ were analyzed by SEM. Arrows indicate ciliated cells and arrowheads indicate shortened cilia. (E) Change in cilia lengths of $\mathrm{Hdac}^{+/ Y}$ and $\mathrm{Hdac}^{-/ Y}$ MTECs exposed to $50 \mathrm{mg} / \mathrm{m}^{3} \mathrm{CS}(n=3$ experiments; 10 SEM images/sample; average cilia length/image was calculated by measuring a minimum of 3 cilia for every multiciliated cell and normalizing to $H d a c 6^{+/ Y}$ untreated control cultures). (F) Number of $\mathrm{Hdac}^{+/ \mathrm{Y}}$ and $\mathrm{Hdac}^{-/ Y} \mathrm{MTECs}$ with cilia longer than $0.6 \mathrm{AU} 24$ hours after exposure to $50 \mathrm{mg} / \mathrm{m}^{3} \mathrm{CS}$ ( $n=3 \mathrm{cultures} / \mathrm{group}$; 5 SEM images/sample normalized to $\mathrm{Hdac}^{+/ Y}$ control cultures) (G) Change in cilia lengths in $\mathrm{Hdac}^{+/ Y}$ and $\mathrm{Hdac}^{-/ Y}$ mice exposed to CS for 2 months ( $n=3-4$ mice/group; $10 \mathrm{H} \& \mathrm{E}$-stained images/mouse; average cilia length/image was calculated by measuring a minimum of 3 cilia in every ciliated cell and normalizing to WT RA controls). All data are the mean \pm SEM. ${ }^{\star} P<0.05,{ }^{* \star} P<0.01$, and ${ }^{* * *} P<0.001$ by one- or two-way ANOVA with Bonferroni's post tests.

be impaired with respect to the CS-induced autophagic response based on decreased lung tissue autophagosome numbers and decreased autophagic flux after CS exposure (as assessed by electron microscopy and lysosomal turnover of LC3B, respectively) (Figure 3, A and B, and refs. 10, 44). Subsequently, we isolated MTECs from Becn $1^{+/-}$mice and subjected them to CS exposure at the ALI. Using SEM to evaluate cilia morphology, we found that WT $\left(\mathrm{Becn}^{+/+}\right)$MTECs sustained greater cilia shortening in response to CS relative to $B e c n 1^{+/-}$MTECs. Quantification of SEM images of WT MTECs confirmed that CS caused a reduction in mean cilia length and reduced the number of epithelial cells with long cilia (using an empirical cutoff of $0.6 \mathrm{AU}$; see Methods). In contrast, Becn $1^{+/}$MTECs were protected from CS-induced cilia shortening (Figure 3, C-E). Similarly, Map1lc3b-/- MTECs, which are deficient in the autophagic protein LC3B, were protected from CS-induced cilia shortening relative to their corresponding WT MTECs (Figure 3, F and G). We also examined the effect of autophagy proteins on cilia length in vivo in mice exposed to CS for 2 months. Becn1 1/- (Figure 3H) or Map1lc36-/- (Figure 3I) mice were resistant to cilia shortening in airway sections relative to their corresponding WT mice after 2 months of CS exposure.

As primary cilia are present on most quiescent cells and are formed in response to starvation (46), we also confirmed the role of beclin 1 in primary cilia regulation using starvation to promote cilia formation in fibroblasts (Supplemental Figure 4A). In this model, primary cilia of Becn $1^{+/-}$fibroblasts were protected from CSE-induced, but not growth factor-induced, primary cilia resorption compared with $B e c n 1^{+/+}$cells, suggesting 
that autophagy plays a critical role in stress-induced primary cilia resorption (Supplemental Figure 4, A and B).

Protection against cilia shortening in Becn $1^{+/-}$MTECs in response to $C S$ is independent of cell death regulation. Although autophagy is a prosurvival response during starvation, we previously reported that increased expression of autophagic proteins was associated with apoptotic cell death in CS-exposure models $(10,11,47)$. We therefore assessed the relative role of cell death in the effects of autophagy on cilia length during CS exposure using WT or Becn1 ${ }^{+/-}$MTECs. Exposure to $50 \mathrm{mg} / \mathrm{m}^{3} \mathrm{CS}$, which caused cilia shortening in MTECs (Figure 1), did not cause loss of cell viability, as measured by TER and LDH release (Supplemental Figure 5, A and B), or apoptosis, as evaluated by SEM and caspase- 3 cleavage (Supplemental Figure 5, C and D) in either WT or Becn ${ }^{+/-}$MTECs. At elevated CS exposure levels $\left(100 \mathrm{mg} / \mathrm{m}^{3} \mathrm{CS}\right)$, however, which cause loss of cell integrity and apoptosis (Supplemental Figure 1), the $\mathrm{Benn}^{+/-}$cultures were protected from injury relative to $\mathrm{Becn} 1^{+/+}$ MTECs, as measured by TER and LDH release (Supplemental Figure 5, A and B), and displayed reduced apoptotic cells and caspase- 3 cleavage relative to Becn $1^{+/+}$MTECs (Supplemental Figure 5D). Similar results were obtained with LC3B knockout (Map1lc3b-/-) MTECs exposed to $100 \mathrm{mg} / \mathrm{m}^{3}$ CS (Supplemental Figure 5, D and E). Interestingly, treatment of MTECs with the general autophagy inhibitor 3-methyladenine (3-MA) attenuated CS-induced caspase- 3 cleavage after $100 \mathrm{mg} / \mathrm{m}^{3}$ CS treatment (Supplemental Figure 5F).

CS modulates the expression of proteins involved in cilia regulation in epithelial cells. In addition to modulation of autophagy as a general mechanism, we next sought to determine whether CS can affect the expression of proteins known to regulate primary cilia length. Using human bronchial epithelial (HBE) cells as a primary screen, we examined the effects of CS on the expression of AurA, HEF1, and HDAC6. HBE cells were treated with 20\% CSE (approximately equivalent to $50 \mathrm{mg} / \mathrm{m}^{3} \mathrm{CS}$ for 24 hours). CSE increased the expression of AurA and HDAC6 in a time-dependent manner (Supplemental Figure 6A). Furthermore, CSE caused a time-dependent increase in the inhibitory phosphorylation of GSK3 $\beta$, a protein involved in primary cilia maintenance (34). Interestingly, CSE increased levels in HBE cells of acetylated $\alpha$-tubulin, a known HDAC6 substrate (Supplemental Figure 6A). CSE had no effect on the expression of HEF1 in HBE cells. Consistent with these findings, exposure to $50 \mathrm{mg} / \mathrm{m}^{3}$ CS caused a time-dependent increase in HDAC6 protein expression in primary MTECs (Supplemental Figure 6B).

$H d a c 6^{-/ Y}$ mouse tracheal epithelial cells, which are deficient in autophagy, were resistant to CS-induced cilia shortening. HDAC6, known to regulate primary cilia resorption, has also been shown to regulate autophagy at the step of autophagosome-lysosome fusion in mouse embryonic fibroblasts (38). We therefore sought to determine whether changes in HDAC6 expression and the associated regulation of autophagy could regulate airway epithelial cilia length in vitro and in vivo. We first examined the regulation of HDAC6 in the airways of mice after CS exposure. Importantly, in vivo analysis of airway epithelia revealed that HDAC6 localized to the apical cell surface and costained with the cilia marker acetylated $\alpha$-tubulin (Figure 4A). We then chose to further study the functional role of HDAC6 in ciliary responses to CS exposure with a loss-of-function approach using mice deficient in HDAC6 expression $\left(H d a c 6^{-/ Y}\right)$. We determined that $H d a c 6^{-/ Y}$ mice were autophagy deficient, as shown by reduced autophagosome formation (Figure 4B) and decreased autophagic flux in response to CS exposure (Figure 4C). We isolated MTECs from $\mathrm{Hdac}^{-/ Y}$ mice and exposed them to CS in vitro. SEM analysis revealed that $H d a c \sigma^{-/ Y}$ MTECs were resistant to cilia shortening in response to CS compared with the WT $\mathrm{Hdac}^{+/ Y}$ cultures (Figure 4D). CS caused a reduction in mean cilia length and reduced the number of epithelial cells with long cilia (using a cutoff of $0.6 \mathrm{AU}$ ) in WT cells. In contrast, $H$ dac $^{-/ Y}$ MTECs were protected from CS-induced cilia shortening (Figure 4F). Hdac6 ${ }^{-/ Y}$ mice were resistant to cilia shortening in the airways after exposure to CS in vivo for 2 months, as determined by quantification of cilia from $\mathrm{H} \& \mathrm{E}$ images of large airways (Figure 4G).

We also examined the role of HDAC6 in CS-induced epithelial cell death. Similar to observations using Becn $1^{+/-}$MTECs, $H$ dac $^{-/ Y}$ cultures displayed no differential phenotype relative to WT cultures with respect to cell death at $50 \mathrm{mg} / \mathrm{m}^{3} \mathrm{CS}$. In response to $100 \mathrm{mg} / \mathrm{m}^{3} \mathrm{CS}$ exposure, $H d a c 6^{-/ Y}$ cultures were protected from injury, as measured by LDH release (Supplemental Figure 7A), and exhibited reduced apoptotic cells, as determined by SEM and caspase-3 cleavage relative to $\mathrm{Hdac}^{+/ Y}$ MTECs (Supplemental Figure 7, B and C). Taken together, these results indicate that in addition to core autophagic proteins (e.g., beclin 1), the cytosolic deacetylase HDAC6 plays a role in cilia shortening in the airways in response to CS.

CS-induced autophagic processing of ubiquitinated proteins is regulated by HDAC6. In addition to the regulation of autophagy, HDAC6 is also implicated in the efficient autophagic processing of ubiquitinated protein aggregates in diverse models, including mouse embryonic fibroblasts, human A549 and 293T cells, and Drosophila melanogaster $(36,37,48,49)$. We therefore assessed the role of HDAC6 in protein aggregation and clearance after CS exposure. CS, a potent prooxidant agent, is known to alter protein homeostasis by causing the oxidative modification of proteins, the disruption of protein folding, as well as altered protein processing in the ER, leading to the formation of protein aggregates (7-9). Consequently, protein aggregates may be solubilized by protein chaperones and removed by proteosomal degradation, or organized in centralized aggresomes and removed by the autophagic pathway (50). Consistently, we observed that CS induced global protein ubiquitination in WT MTECs (Figure 5A). In contrast, MTECs deficient in HDAC6 (Hdac6 $6^{-/ Y}$ ) displayed increased levels of protein ubiquitination under basal conditions as well as after CS exposure relative to WT MTECs (Figure 5A). Staining of WT MTECs with a stain specific for aggregated protein revealed that CS exposure causes the formation of large perinuclear cytosolic aggregates (aggresomes) in ciliated cells (Figure 5B and Supplemental Figure 8A). Conversely, Hdac6 ${ }^{-/ Y}$ ciliated MTECs displayed increased diffuse cytosolic staining for protein aggregation without evidence of aggresome formation (Figure 5B and Supplemental Figure 8A).

The incidence of protein aggregate puncta induced by CS in MTECs could be further augmented by treatment with CQ (Figure 5C). Similar results were observed in cultured epithelial cells in which CQ treatment increased the steady-state levels of ubiquitinated protein to a greater extent in CSE-exposed cells relative to control cells (Supplemental Figure 8B). Confocal analysis revealed that protein aggregates induced by CS colocalized with the autophagosome marker LC3B (Supplemental Figure 8C). These results, taken together, suggest that ubiquitinated protein aggregates induced by CS are cleared in part through HDAC6mediated autophagic pathway processing. 
A

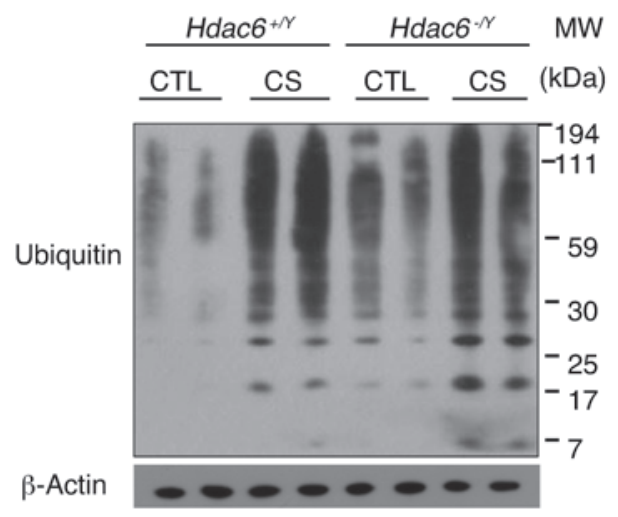

B

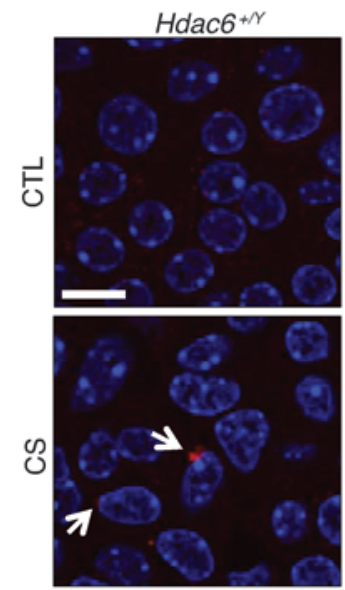

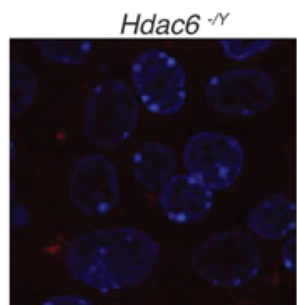

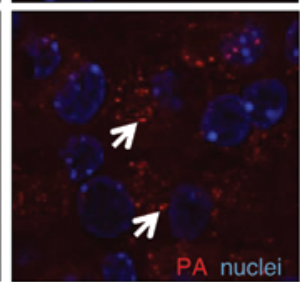

E
D

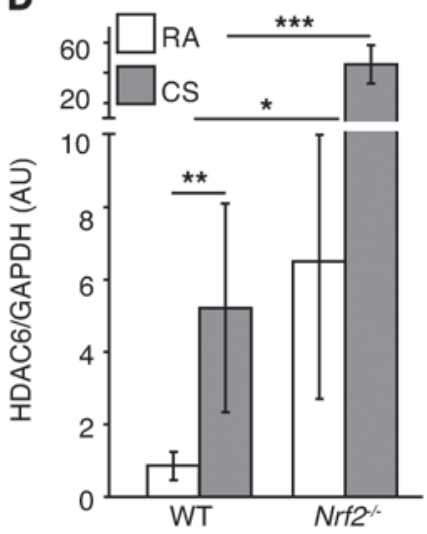

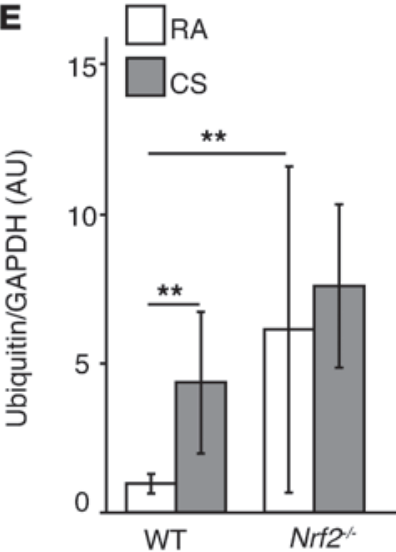

Figure 5

HDAC6 deficiency is associated with impaired autophagic protein clearance. (A) The global ubiquitin pattern was assessed in $H_{d a c 6} 6^{+/ Y}$ and Hdac6 $^{-/ Y}$ MTEC cultures 24 hours after treatment with $50 \mathrm{mg} / \mathrm{m}^{3} \mathrm{CS}$. Samples are presented in duplicate. $\beta$-Actin served as the standard. (B) Protein aggregates (PA; shown in red) were analyzed in MTEC cultures derived from Hdac6 $6^{+/ Y}$ and $H d a c 6^{-/ Y}$ mice 24 hours after exposure to CS $\left(50 \mathrm{mg} / \mathrm{m}^{3}\right)$. Scale bar: $10 \mu \mathrm{m}$ ( $n=3$ MTEC cultures; 5 fields analyzed/culture) (C) Quantification of protein aggregate puncta in MTEC cultures, which were pretreated with $50 \mathrm{mg} / \mathrm{m}^{3} \mathrm{CS}$ followed 15 minutes later by CQ $(25 \mu \mathrm{m})$ in the basal media and harvested 2 hours later ( $n=2-3$ MTEC cultures; 3-4 fields analyzed per culture). (D) Nrf2-l- and WT mice ( $n=4$ mice/group) were exposed to RA or CS for 2 weeks. (E) Global protein ubiquitination was quantified by densitometry in $\mathrm{Nrf2}^{-/-}$and WT mouse lungs after exposure to RA or CS ( $n=2$ mice/group). All data are the mean \pm SEM. ${ }^{\star} P<0.05$ by an unpaired Student's $t$ test $(\mathbf{C})$, and ${ }^{\star} P<0.05,{ }^{\star \star} P<0.01$, and ${ }^{\star \star \star} P<0.001$ by one- or two-way ANOVA and Bonferroni's post tests ( $\mathbf{D}$ and $\mathbf{E})$.

Nrf2 deficiency increases HDAC6 expression. We hypothesized that HDAC6 may be regulated in response to increased protein ubiquitination. We therefore examined the relationship between protein ubiquitination and HDAC6 expression using a genetic model of susceptibility to oxidative stress (51). Mice genetically deficient in nuclear factor erythroid 2 -related factor ( $\left.\mathrm{rrf2} 2^{-/-}\right)$, a master regulator of the cellular antioxidant response, have been previously shown to display impaired proteasomal activity and heightened ER stress (8) as well as increased susceptibility to airspace enlargement after chronic CS exposure (51). Furthermore, NRF2 expression has been shown to be decreased in the lungs of patients with $\operatorname{COPD}(8,52,53)$. We found that cells isolated from

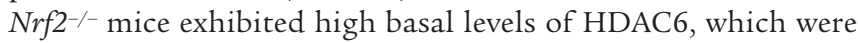
further augmented upon CS exposure (Supplemental Figure 9A). Similarly, $\mathrm{Nrf2}^{-/-}$mice displayed elevated basal expression of HDAC6 in the lungs, which was further increased in response to 2 weeks of CS exposure (Figure 5D). Increased basal and CS- inducible protein ubiquitination was also observed in lungs from $\mathrm{Nrf2}^{-/-}$mice (Figure 5E). CS exposure in mice increased an association between HDAC6 and high molecular weight ubiquitinated protein in mouse lung homogenates (Supplemental Figure 9B). Taken together, these experiments suggest that HDAC6 expression and function are related to the cellular processing of ubiquitinated proteins in CS exposure models.

Sirtuin 1 regulates HDAC6 through acetylation. It has previously been shown that NRF2 is regulated by sirtuin 1 (SIRT1), an $\mathrm{NAD}^{+}$-dependent protein/histone deacetylase that is also reduced in the lungs of patients with $\operatorname{COPD}(54,55)$. We next assessed whether HDAC6 could be regulated by SIRT1-dependent acetylation. The acetylation of HDAC6 was increased in lung homogenates from Sirt $1^{+/}$mice (Supplemental Figure 9C), suggesting that SIRT1 is a deacetylase for HDAC6. HDAC6 expression in lung homogenates was also increased upon CS exposure of Sirt $1^{+/-}$mice (Supplemental Figure 9, C and D). 
Interestingly, the appearance of ubiquitin laddering in response to CS was diminished in Sirt $1^{+/-}$mice (Supplemental Figure 9E). These results suggest a relationship between the regulation of HDAC6 expression and acetylation state and ubiquitinated protein clearance in the lung.

Cilia components may be selective substrates for ubiquitin-targeted autophagic clearance. Next, we investigated whether CS exposure promotes the degradation of cilia components through ubiquitination and processing by the autophagic pathway. We observed that CS induced the dose- and time-dependent polyubiquitination of the ciliary protein IFT88 in MTECs, as evidenced by the formation of immunoreactive high-molecular-weight bands after treatment with $100 \mathrm{mg} / \mathrm{m}^{3} \mathrm{CS}$ (Supplemental Figure 10A). To investigate whether CS causes the degradation of cilia components by autophagy, we first assessed the colocalization of cilia with autophagy markers. By confocal immunofluorescence analysis, we found that $\mathrm{CS}\left(50 \mathrm{mg} / \mathrm{m}^{3}\right)$ induced the colocalization of acetylated $\alpha$-tubulin and LC3B in MTECs (Figure 6A). At a higher concentration of CS $\left(100 \mathrm{mg} / \mathrm{m}^{3}\right)$, there was evidence of cilia loss, with acetylated $\alpha$-tubulin staining occurring mainly in the cytoplasm (Figure 6A).

Furthermore, in MTECs subjected to CS exposure $\left(100 \mathrm{mg} / \mathrm{m}^{3}\right)$, we detected autophagosomes containing the ciliary protein IFT88 in proximity to basal bodies by immunogold TEM analysis (Figure 6B). We observed by subcellular fractionation that IFT88 and the ciliary protein centrin 1 accumulated in the lysosome-enriched fraction following CS exposure (50-100 mg/m $\mathrm{m}^{3}$ ) (Figure 6C).

To validate these observations in vivo, we subjected LC3B-GFP mice to 3 weeks of RA or CS exposure with or without the autophagic inhibitor leupeptin to assess whether ciliary proteins localized to autophagosomes. GFP immunoprecipitates from autophagosome/lysosome-enriched lung homogenates (see Methods) were subjected to Western blot analysis for the detection of the ciliary proteins IFT88, ARL13, centrin 1, and pericentrin (Figure 6D). CS exposure significantly increased the amount of ARL13, IFT88, centrin 1 , and pericentrin 1 localized to autophagosomes in mouse airways (Figure 6D). LC3B expression was increased in response to CS and leupeptin administration, whereas HDAC6 localization to autophagosomes was only increased in the CS-exposed samples (Supplemental Figure 11A). The localization of ciliary proteins to autophagosomes was also increased by leupeptin injection, suggesting that these proteins may be basally turned over by autophagy (Figure 6D). Finally, a combination of leupeptin administration and CS exposure resulted in maximal detection of these proteins in autophagosomes (Figure 6D). Taken together, these observations suggest that the autophagic pathway may contribute to the turnover of cilia components during CS-induced oxidative stress.

Therapeutic targeting of the HDAC6-dependent autophagic pathway in vivo and chemical chaperones rescue MCC dysfunction during CS exposure. Our observations, taken together, suggest that CS perturbs proteostasis and subsequently causes cilia dysfunction in a manner dependent on HDAC6 and on activation of the autophagic pathway. We therefore sought to determine whether modulation of these individual components could be exploited for therapeutic gain in a physiological model of airway function.

We next assessed the effects of CS on mucociliary function in mice. We used a $3 \mathrm{D}$ whole-mouse $\mu$ SPECT imaging approach to assess MCC with an oropharyngeal aspiration procedure (56), in which the radiopharmaceutical ${ }^{99 \mathrm{~m}}$ technetium-sulfur colloid $\left({ }^{99 \mathrm{~m}} \mathrm{Tc}-\mathrm{sc}\right)$ was instilled into mouse lungs (Figure 7, A and B, and Supplemental
Video 1). Using this assay, the efficacy of ${ }^{99 \mathrm{~m}} \mathrm{Tc}$-sc clearance from mice lungs exposed to CS for 3 weeks was significantly reduced compared with that of the air-exposed controls $(P=0.003)$ (Figure 7, $\mathrm{B}$ and $\mathrm{C}$ ), indicative of impaired MCC.

Mice deficient in the autophagic protein beclin 1 were protected from CS-impaired MCC $(P=0.003, P=0.0007)$ (Figure 7C). Similar results were observed with autophagy-deficient LC3B knockout mice (Map1lc36-/-) (Figure 7C). In contrast, $\mathrm{Nrf2} \mathrm{2}^{-/-}$mice had dramatically impaired basal MCC compared with exposure of WT mice to RA $(P=0.02)$ (Figure $7 \mathrm{C})$. Nrf2 $2^{-/}$mice exposed to CS had no MCC after 3 weeks of exposure (Figure 7C). These experiments suggest that autophagy deficiency may confer protection against aberrant airway responses to CS in vivo, whereas deficiency in the antioxidant response aggravates these responses.

Consistent with our in vitro findings that $\mathrm{Hdac}^{-/ Y}$ MTECs displayed a cilia protection phenotype similar to that in $\mathrm{BeCn} 1^{+/}$ MTECs, we observed that the $H \operatorname{dac}^{-/ Y}$ mice were also protected from CS-induced MCC disruption $(P=0.007)$ (Figure $7 C)$. In addition, mice coadministered with the selective HDAC6 deacetylase chemical inhibitor tubastatin A were protected from CSimpaired MCC $(P=0.01)$ (Figure 7D). These experiments, taken together, suggest that HDAC6 inhibition improves ciliated airway function during CS exposure.

We hypothesized that protecting epithelial cell proteostasis by enhancing protein folding in response to CS may protect ciliated epithelial cells from cilia shortening. The chemical chaperone 4-phenyl butyric acid (4-PBA) has been previously shown to influence protein folding, protect against oxidative stress, and enhance the clearance of protein aggregates in vivo $(57,58)$. Administration of 4-PBA for the duration of CS exposure also prevented CS-impaired MCC $(P=0.007)$ (Figure 7D). 4-PBA was also found to protect against CS-induced protein ubiquitination in vivo (S.M. Cloonan, unpublished observations). These data suggest a coordinate role for HDAC6, autophagy, and altered proteostasis in MCC dysfunction in vivo, which can be prevented by pharmacological agents targeting these pathways.

$C S$-dependent inhibition of MCC was reversible and did not involve apoptosis in vivo. We next evaluated the reversibility of CS-induced impairment of MCC. Mice ( $n=5$ per group) were exposed to CS for 3 weeks and left to recover after smoking cessation for 1 or 3 weeks before measuring in vivo MCC. One week of recovery restored MCC to $80 \%$ of the levels observed in the RA controls (Figure 7E). Mice that had 3 weeks to recover showed nearly complete recovery from CS-impaired MCC (Figure 7E). These experiments demonstrate that loss of MCC induced by CS exposure is reversible. Assessment of H\&E-stained lung sections of mice exposed to RA or CS for 3 weeks, or for 3 weeks followed by 1 week recovery, showed no evidence of basal cell proliferation or squamous metaplasia (Supplemental Figure 12).

We also assessed the relative role of apoptosis during in vivo CS exposure. C57Bl/6 mice were subjected to CS exposure in vivo for the indicated times. TUNEL staining of lung tissue sections revealed minimal changes in TUNEL-positive cells in the airways or parenchyma (Figure 7F). In contrast, robust TUNEL-positive staining was observed in airways and parenchymal lung tissue after acute hyperoxia exposure $\left(95 \% \mathrm{O}_{2}\right.$ for 72 hours), as a positive control of apoptosis. Becn $1^{+/-}$and $H d a c 6^{-/ Y}$ showed no significant differences in TUNEL staining after 2 months of CS exposure, comparable to that observed in WT mice (Figure 7F). 

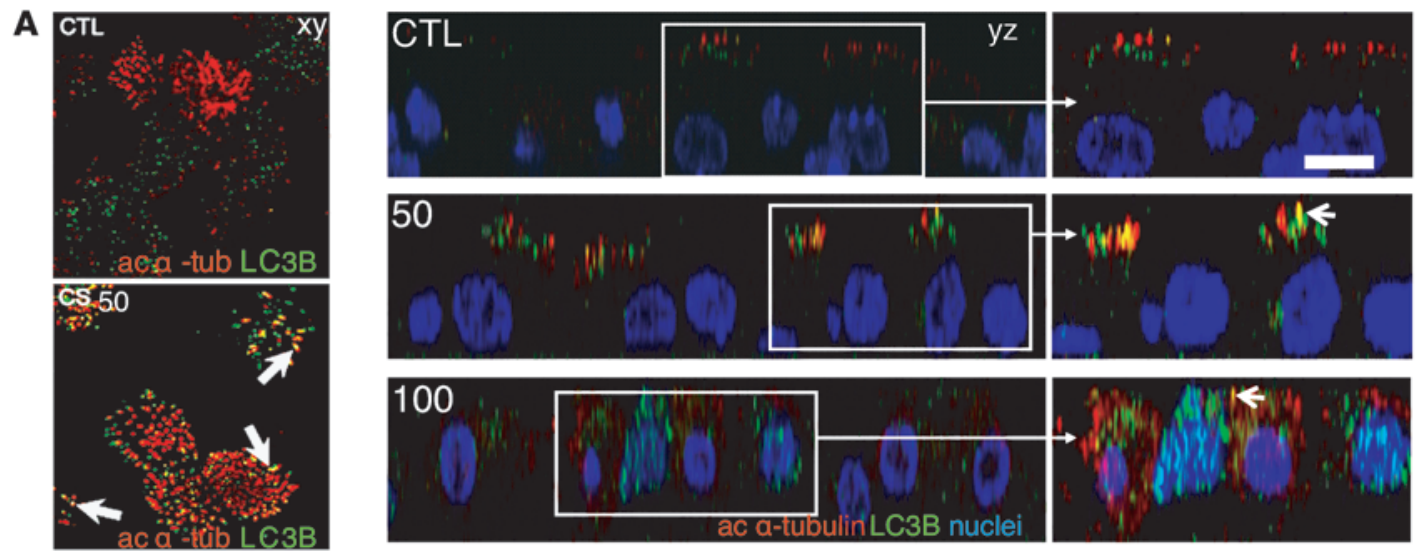

B

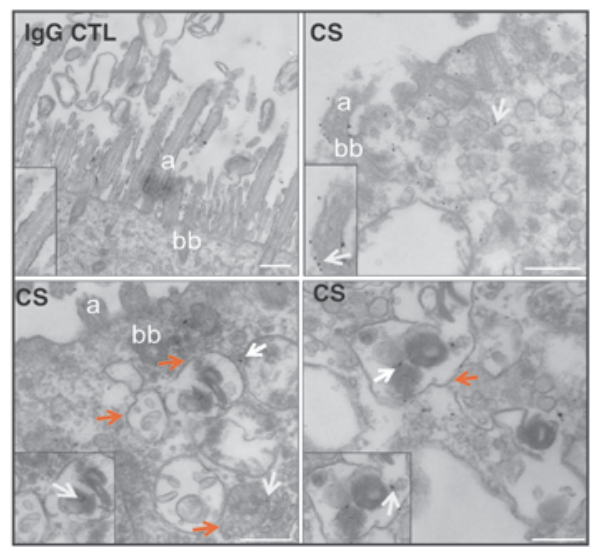

C
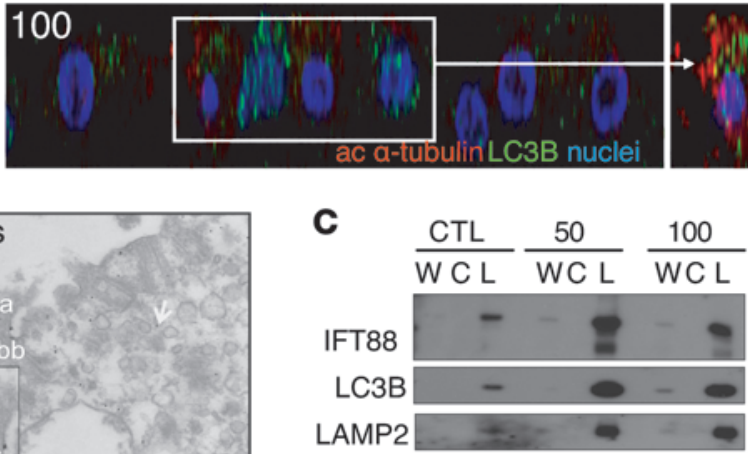

$\frac{C T L}{W C L} \frac{50}{W C L} \frac{100}{W C L}$

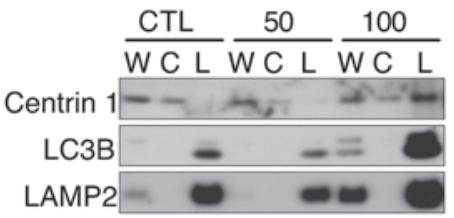

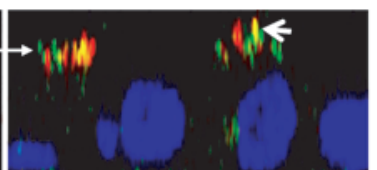

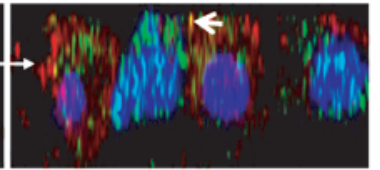

D

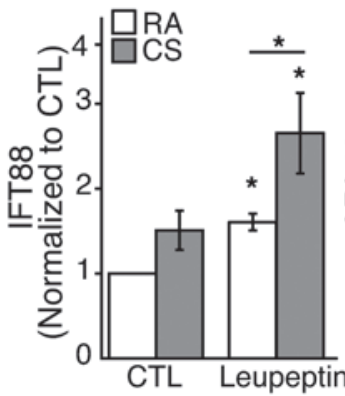

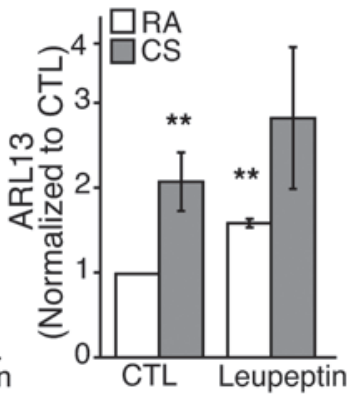
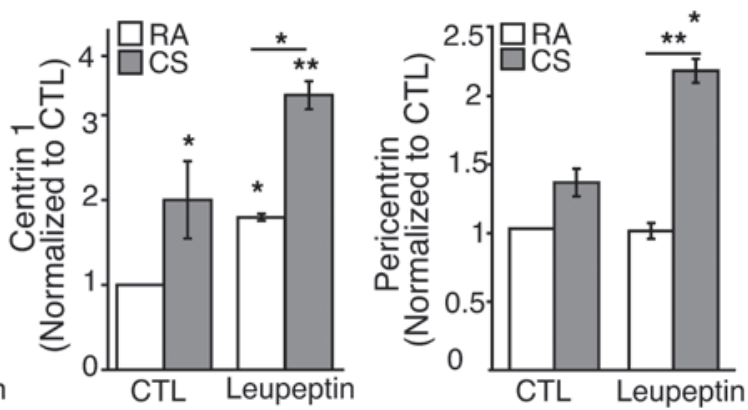

Figure 6

Ciliary proteins may be substrates of autophagy in response to CS. (A) $x y$ and $y z$ confocal analysis of acetylated $\alpha$-tubulin, Hoechst staining, and LC3B showing colocalization (arrows) in MTECs 24 hours after CS $\left(50 \mathrm{mg} / \mathrm{m}^{3}\right)$ exposure. At a higher dose of CS (100 mg/m $\left.{ }^{3}\right), \alpha$-tubulin localized primarily to the cytosol, with evidence of cilia loss. Data are representative of two experiments. ac, acetylated. (B) Ciliary protein IFT88 or IgG CTLs were immunogold labeled (white arrows) in ciliated MTECs 24 hours after CS $\left(100 \mathrm{mg} / \mathrm{m}^{3}\right)$ exposure (insets demonstrate staining specificity; original magnification of insets, $\times 4$ ). Red arrows indicate autophagosomes. (C) MTEC homogenates were fractionated into cytoplasmic-enriched $(C)$ and lysosome-enriched (L) fractions from whole-cell lysates (W) 24 hours after treatment with CS at the indicated dose. Figures are representative of one to three independent determinations. Fractions were immunoblotted with IFT88, centrin 1, and LC3B. LAMP2 served as a lysosomal marker. IFT88 and centrin 1 densitometry (right). (D) Autophagosome/lysosome-enriched fractions of lungs from LC3B-GFP mice exposed to RA $(n=8)$ or CS $(n=9)$ for 3 weeks and immunoprecipitated with anti-GFP. Mice were injected with PBS $(n=4)$ or leupeptin $(n=4)$ for 2 hours prior to harvesting. GFP-immunoprecipitated LC3B-positive fractions were immunoblotted for the cilia markers IFT88, ARL13, centrin 1, and pericentrin. Bar charts are representative of three to four immunoblots normalized to RA PBS controls. Scale bars: $10 \mu \mathrm{m}$. ${ }^{*} P<0.05$ and ${ }^{* \star} P<0.01$ by an unpaired Student's $t$ test and one- or two-way ANOVA and Bonferroni's post tests.

Identification of HDAC6 as a novel regulated gene in COPD. Since our experimental observations indicated that HDAC 6 may play a detrimental role in ciliated epithelial cell responses to CS exposure in vitro and in vivo, we examined the hypothesis that HDAC6 could be regulated in human COPD. We conducted a genome-wide survey of regions differentially methylated in COPD patients and controls from the Lung Genomics Research Consortium (LGRC; http://lung-genomics.org/research) data. Analysis of these data identified a hypomethylated region upstream of the HDAC6 transcription start site (Figure 8, A-C). 

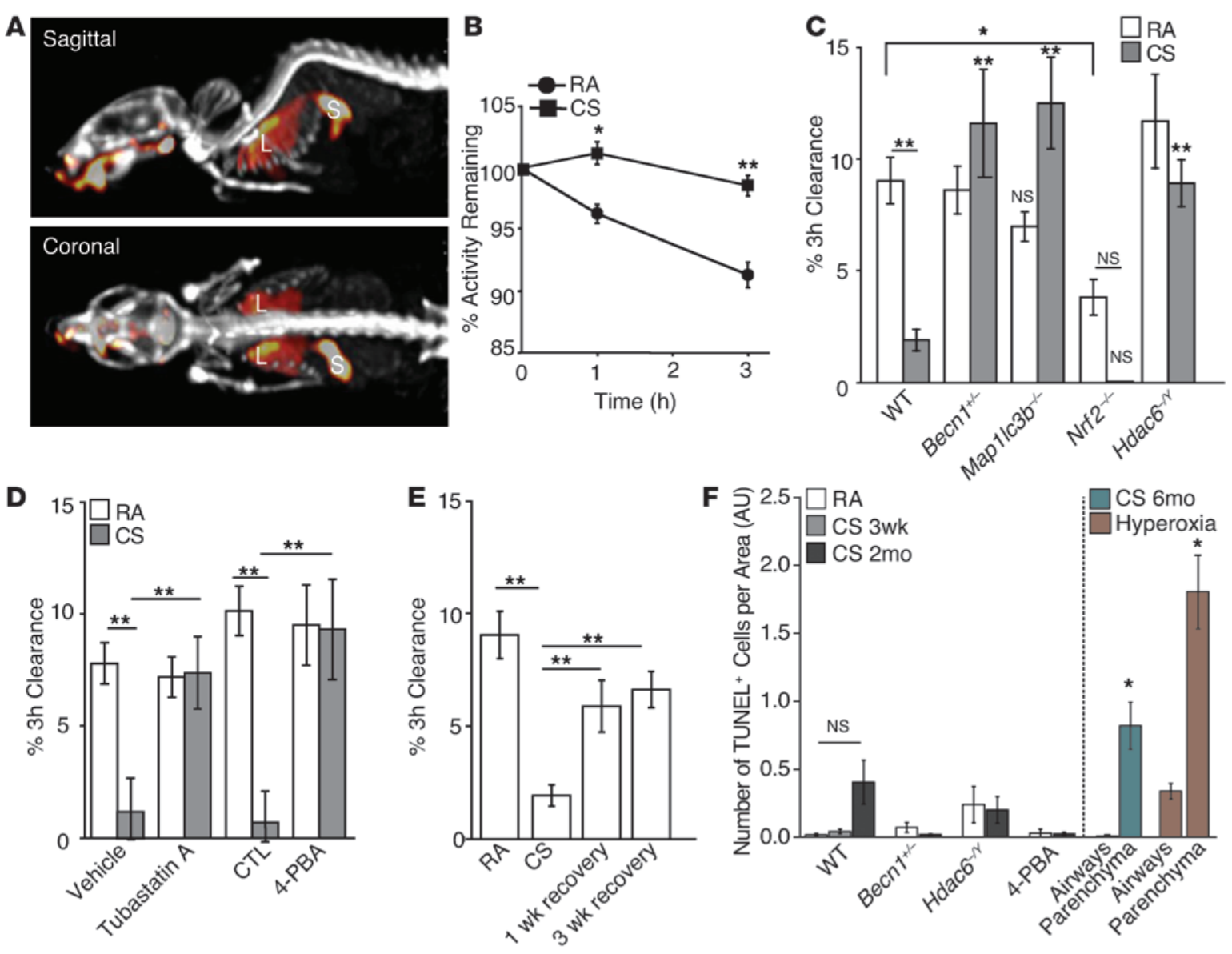

Figure 7

Targeting of MCC function by inhibition of HDAC6 or the autophagic pathway. (A) Image of 99mTc-sc instilled into mouse lungs with skeleton visualized using $99 \mathrm{mT}$ Tc-mdp. Heatmap shows the distribution of $99 \mathrm{mTC}-\mathrm{sc}$ in the lungs (L) and stomach (S). (B) $99 \mathrm{mTc}$-sc in the lungs was quantified from images at $t=0,1$, and 3 hours in mice exposed to RA $(n=5)$ or CS $(n=5)$ for 3 weeks. (C) MCC was assessed after a 3-week exposure to RA or CS in Becn1+/- ( $n=4 /$ group), Map1/c3b-/- $\left(n=5-6 /\right.$ group), Nrf2-/- $\left(n=3-4 /\right.$ group), or Hdac6 ${ }^{+/ Y}$ ( $n=3-4 /$ group) mice. (D) MCC was also assessed in mice coadministered with tubastatin A ( $n=4 /$ group) or with 4-PBA ( $n=3-4 /$ group) and a 3-week exposure to RA or CS. (E) MCC in mice exposed to RA or CS for 3 weeks ( $n=10-13 /$ group) or in mice exposed to CS for 3 weeks followed by a 1- or 3-week recovery period ( $n=5$ /group). (F) TUNEL-positive cells in airways or parenchyma of WT mice ( $n=3 /$ group) exposed to RA or CS for 3 weeks, 2 months, or 6 months; in $\mathrm{HDAC6}^{-/ Y}$ or Becn1+/- mice exposed to CS for 2 months; or in mice treated with 4-PBA and exposed to 3 weeks of CS. Mice were exposed to hyperoxia or the corresponding RA control for 72 hours (far right) (7-10 images/mouse). All data are the mean $\pm S E M$. (B) ${ }^{*} P<0.05$, ${ }^{\star *} P<0.01$ by an unpaired Student's $t$ test. $(\mathbf{C}-\mathbf{F}){ }^{\star \star} P<0.01$ by one- or two-way ANOVA with Bonferroni's post tests.

Using the comprehensive high-throughput arrays for relative methylation (CHARM) platform (59), we profiled methylation at 2,162,405 sites across the genome in 194 lung samples (124 COPD patient samples, 43 controls). We identified differentially methylated regions by fitting linear regressions on logit-transformed percentages of methylation, adjusting for age, sex, and pack-years of smoking and combined $P$ values to define differentially methylated regions $(60,61)$.

We analyzed differential HDAC6 methylation by comparing never-smoker controls, ex-smoker controls, and ex-smokers with COPD (124 ex-smoker COPD patients, 25 ex-smoker controls, and 18 never-smoker controls). Using this analysis, the difference between COPD and control ex-smokers was significant $(P<0.01)$, but the difference between ex- and never-smokers was not significant $(P=0.5)$ (Figure 8A). HDAC6 occurs on chromosome $\mathrm{X}$, which is subject to lyonization. Males and females may have differ- ent baseline levels of methylation. We therefore analyzed data by gender (Figure 8B). From this analysis, we demonstrate that the trend in males is more pronounced, with decreasing methylation from never-smoker controls, to ex-smoker controls $(P<0.01)$, to ex-smoker COPD patients $(P<0.05)$. The difference between the control groups in females was not significant $(P=0.8)$.

Of 6,539 differentially methylated regions, the HDAC6 hypomethylated region is particularly likely to be functional, as it is one of the few differentially methylated regions that overlap human-mouse-rat evolutionarily conserved transcription factorbinding sites (Figure 8C). Hypomethylation of a gene is necessary, but not sufficient, for expression and can be considered to possess an increased potential for expression as compared with a hypermethylated gene (62).

We therefore assessed the expression of HDAC6 in lung tissue obtained from COPD patients (see Supplemental Table 1 for 
A

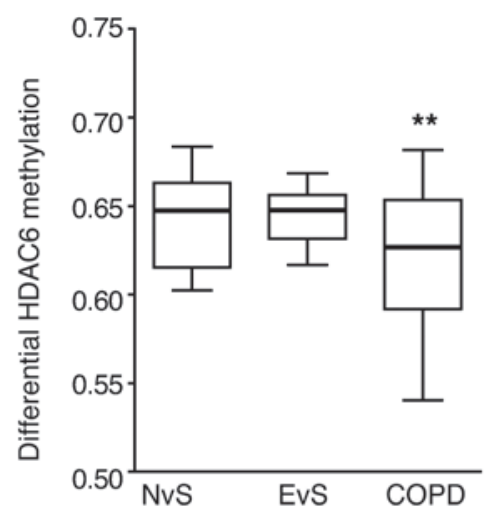

B

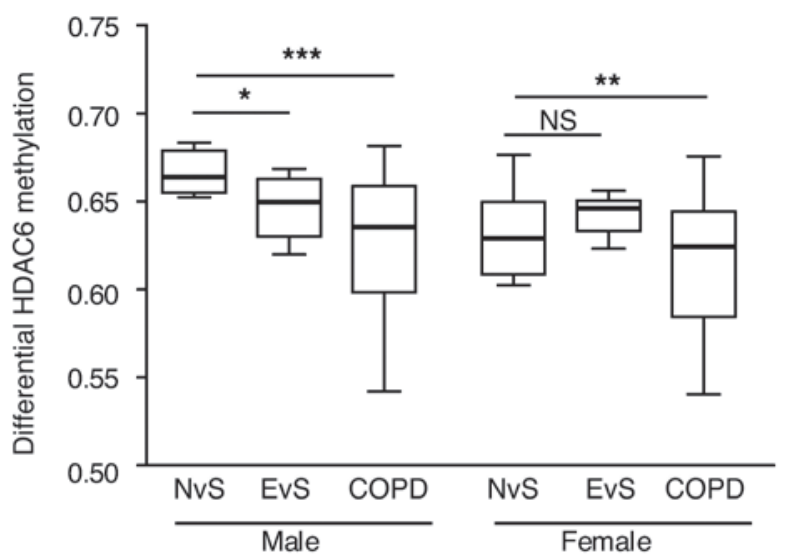

C $\operatorname{chrX}($ p11.23)

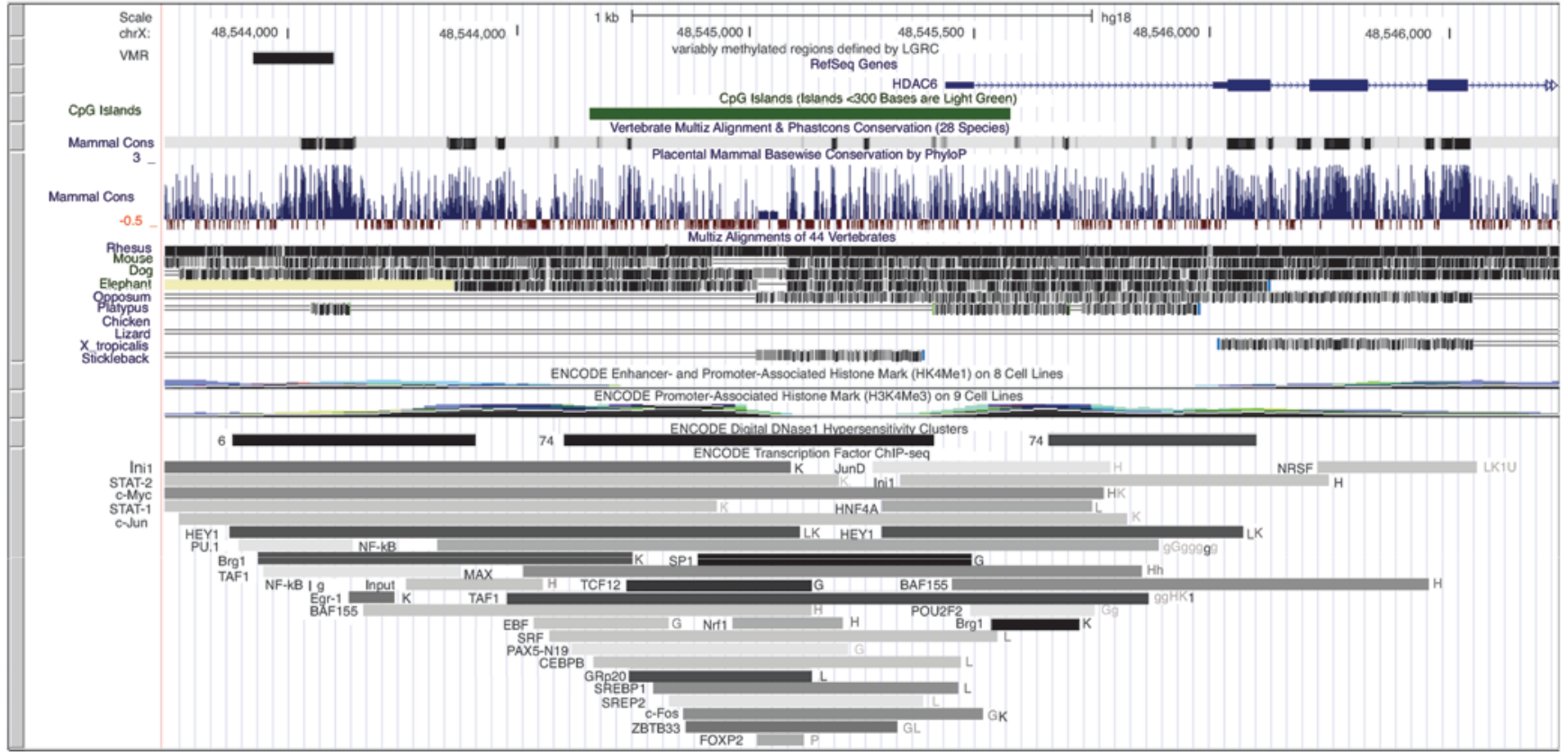

Figure 8

Methylation potentially regulates HDAC6 expression in COPD. (A) Box plot illustrates hypomethylation of a conserved transcription factor-binding site upstream of the HDAC6 gene. Shown here is the unadjusted methylation percentage of never-smokers (NvS) $(n=18)$, ever-smokers (EvS) $(n=25)$, and COPD patients $(n=124)$. The differential methylation analysis used a logit-transformed percentage of methylation and adjusted for age, sex, and pack-years of smoking. (B) Box plot illustrates stratification of $\mathbf{A}$ by sex (NvS males = 6, EvS males $=12$, COPD males $=70$, NvS females $=12$, EvS females = 13, COPD females = 54). (C) The hypomethylated site (labeled "VMR" for variably methylated region) is visualized in the UCSC Genome Browser. The methylated site sits at a CpG "shore," just outside of a CpG island, and overlaps a large conserved region, defined by human-mouse-rat alignment ("Mammal Cons" track). The region is also a binding site for many transcription factors such as STAT1/2, c-Myc, c-Jun, and a DNAsel hypersensitivity site, defined by the ENCODE project. Never-smokers denote patients with no smoking history, eversmokers are patients with a prior smoking history, and COPD patients are those diagnosed with COPD. ${ }^{*} P<0.05,{ }^{\star \star} P<0.01$, and ${ }^{* \star \star} P<0.001$ by one-way ANOVA with Bonferroni's post tests.

patient demographics). HDAC6 was upregulated in lung tissue homogenates of smokers and COPD patients (Figure 9A and Supplemental Figure 13A). We observed increased HDAC6-positive staining in airway epithelia of smokers and COPD patients relative to control subjects (Figure 9B). Interestingly, the expression of the cilia regulatory proteins AurA and phospho-GSK3 $\beta$ were also upregulated in human COPD lung tissue (Supplemental Figure 13B). Taken together, these data suggest that HDAC6 is hypomethylated in ex-smokers, which correlates with an increase in HDAC6 protein expression in ever-smoker controls and COPD patients (ex-smokers) (Figure 9A).

Consistent with human data, we also observed elevated HDAC6 expression in the airways of mice exposed to chronic CS (6 months) (Supplemental Figure 14). Human lung COPD tissues (Supplemental Table 1) displayed increased accumulation of aggregates and protein inclusion bodies in ciliated cells of 
A

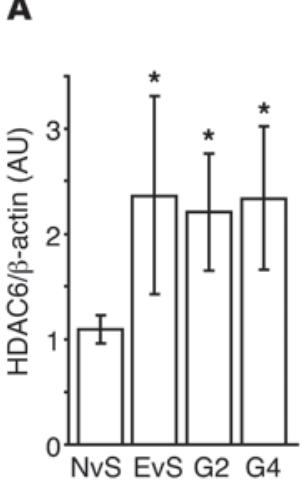

B
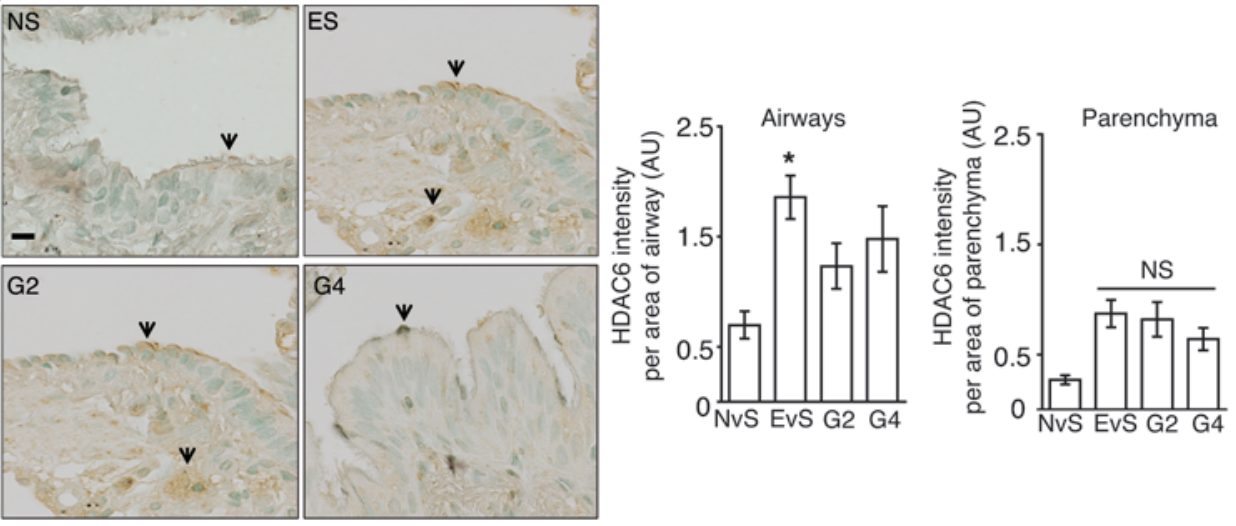

C

NvS

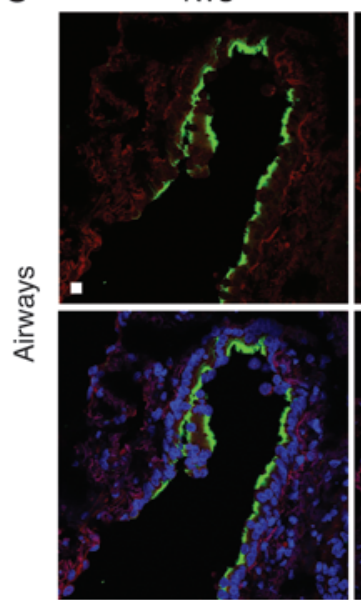

EvS
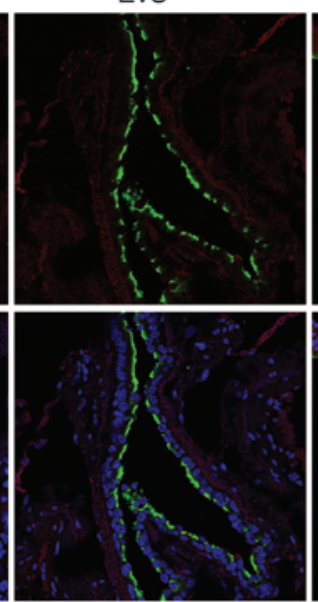

G2

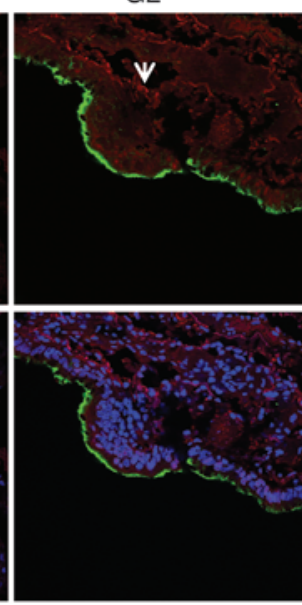

G4

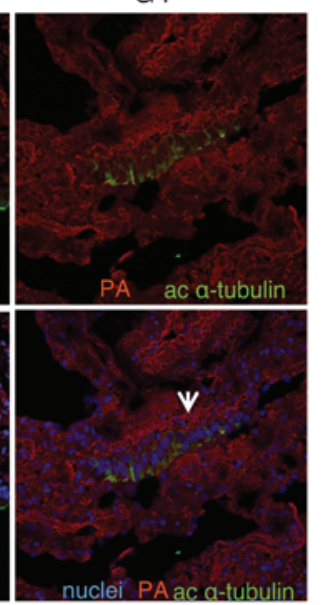

Figure 9

HDAC6 expression is regulated in COPD and during CS exposure. (A and B) HDAC6 expression was assessed in COPD patients. (A) HDAC6 expression was assessed by densitometric analysis of immunoblots of never smoker, ever-smoker, and COPD patient lung tissues (G2, G4) ( $n=5$ patients/group). Band intensities were normalized to $\beta$-actin. (B) Representative immunostaining from airway tissues of never-smokers ( $n=2$ patients, 5 images/patient), ever-smokers ( $n=4$ patients, 3-5 images/patient), and COPD patient lung tissue (G2, G4) ( $n=2-3$ patients, 3-5 images/patient) stained with HDAC6 and quantified by measuring the intensity of HDAC6 staining by threshold analysis using ImageJ. Arrows indicate HDAC6 staining. (C) Protein aggregates (PA, shown in red) were observed in the airways (ciliated airway epithelial cells identified by green acetylated $\alpha$-tubulin staining) of human lung tissue. Never-smokers denote patients with no smoking history, ever-smokers are patients with a prior smoking history, and COPD patients are those with GOLD stage 2 (G2) and 4 (G4) disease severity. Scale bars: $10 \mu \mathrm{m}$. Arrows indicate protein aggregates and inclusion bodies. All data are the mean \pm SEM. ${ }^{*} P<0.05$ by one-way ANOVA followed by a Bonferroni's post test.

the airways (Figure 9C) and in the parenchyma (Supplemental Figure 15), which correlated with disease severity (classified by the Global Initiative for Obstructive Lung Disease [GOLD] scale of increasing severity from $0-4$ ).

\section{Discussion}

Autophagy may influence the progression of human diseases by playing multifunctional roles in the regulation of organelle homeostasis, protein turnover, inflammation, and innate and adaptive immune responses (63). Previous work from our laboratory has identified a candidate role for autophagy in the pathogenesis of COPD $(10,11)$. We have observed enhanced autophagic proteins and autophagosome numbers in human clinical samples of COPD (10). Furthermore, we found that genetic deletion of specific autophagic proteins inhibits emphysema development in a mouse model of chronic CS exposure $(10,11)$. Our mechanistic studies have further elucidated that autophagic proteins can regulate epithelial cell death in response to CS exposure through interactions with apoptotic proteins, in particular, by activating the extrinsic apoptotic pathway $(10,11,47)$.

CS is known to adversely impact cilia length, number, and function in the airways $(64,65)$. The resulting disruption in mucociliary function may render the host susceptible to infection and particle-induced lung injury, which in turn may contribute to the pathogenesis of chronic bronchitis and emphysema $(3,4,25,27)$. Although it is well established that CS exposure causes cilia shortening $(25,27)$, the underlying cellular and molecular mechanisms for this phenomenon remain incompletely delineated. In this study, we demonstrate for what we believe to be the first time that CS can induce cilia shortening through an autophagy-dependent mechanism mediated by HDAC6, a process we term "ciliophagy" (Figure 10). 


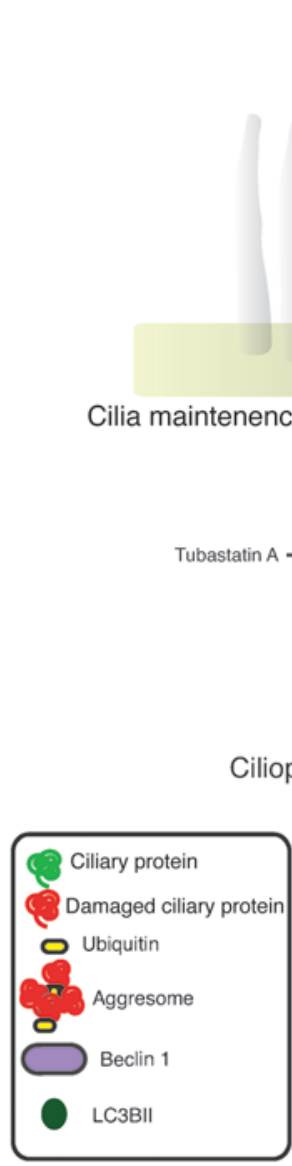

Homeostasis
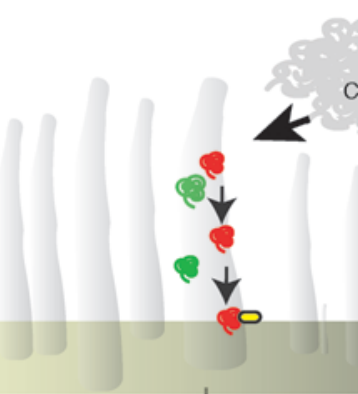

\section{CS}
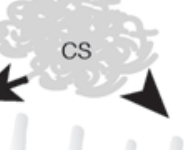

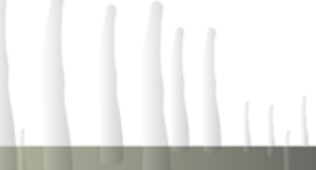
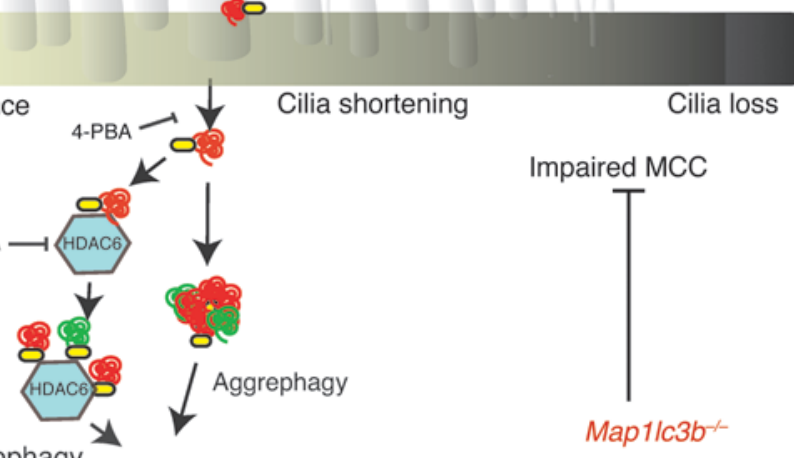

Ciliophagy

Map1/c3b-

Becn1+-

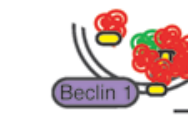

Phagophore
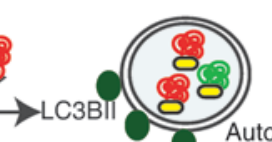

$\mathrm{Hdac6}^{-\gamma}$

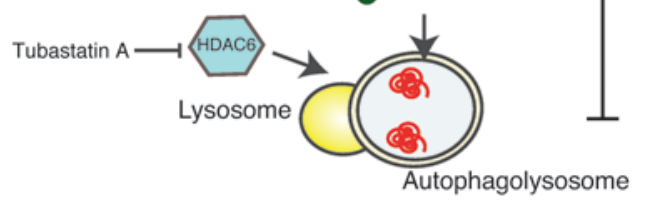

Maladaptation

Epithelial cell death

\section{Figure 10}

Schematic of ciliophagy. CS induces oxidative stress, which leads to ciliary protein damage, misfolding, ubiquitination, and the formation of intracellular protein aggregates. HDAC6 recognizes ubiquitinated protein aggregates and delivers them to the autophagosome, a process dependent on the autophagy proteins LC3B and beclin 1. Ciliary proteins are delivered to the lysosome for degradation or recycling. In cases of chronic oxidative stress, ciliary proteins are degraded, resulting in a shortening of airway cilia that contributes to impaired MCC. Genetic deletion of LC3B (Map1/c3b), beclin 1 (Becn1), or HDAC6 (Hdac6), or inhibition of HDAC6 (tubastatin A) or protein misfolding (4-PBA) alleviates CS-induced impairment of MCC. Severe oxidative stress eventually leads to programmed epithelial cell death, a process that may involve excessive autophagy.
Once thought to represent a process for bulk assimilation of cytoplasm, increasing evidence suggests that autophagy uses highly selective mechanisms for the delivery of cargo to autophagosomes. These include mitophagy, aggrephagy, and xenophagy (the selective degradation of mitochondria, protein aggregates, and pathogens, respectively) $(14,21-24)$. In the current study, we identified cilia components as substrates for autophagic pathway processing during CS exposure. We observed by immunofluorescence and immunogold electron microscopy that cilia components colocalize with autophagosomes. Furthermore, we show that ciliary proteins are enriched in autophagosomal/lysosomal fractions upon CS exposure in vitro and in vivo, suggesting that ciliary proteins may be sequestered by autophagy in response to CS exposure. Our data collectively suggest that the increased activation of the autophagic pathway regulates cilia length and dysfunction during CS exposure. In this model, increased morphological and biochemical indicators of autophagy correlated with cilia shortening. Furthermore, short-term exposure to CS in vitro, or subchronic exposure in vivo, resulted in apparent increases in the autophagic processing of substrate proteins (increased flux) in MTECs and lung tissue, respectively. Mice with genetic deletion of autophagic proteins $\left(B e c n 1^{+/-}\right.$and Map1lc3b-/-) were resistant to cilia shortening caused by CS exposure in vitro and in vivo. These data may be supported by previous studies that link cilia regulation to autophagy. Specifically, knockout of Atg7 in the airways was shown to disrupt cilia formation (66). Hyperactivation of mammalian target of rapamycin (mTOR), an inhibitor of autophagy, resulted in abnormally long cilia (67), whereas rapamycin, an activator of autophagy, was recently reported to shorten embryonic cilia (68).

Evaluation of the expression levels of proteins known to regulate primary cilia length in human bronchial epithelial cells subjected to CS treatment revealed several candidate molecular targets. CS increased HDAC6 in a time-dependent manner in HBE cells and MTEC cultures. CS also increased the expression of AurA, a protein that induces primary ciliary disassembly (shortening) by activating HDAC6 (33). CS induced the phosphorylation of GSK3 $\beta$, a modification that inhibits GSK3 $\beta$ activity and leads to loss of primary cilia (34). Furthermore, AurA, HDAC6, and phospho-GSK3 $\beta$ were upregulated in human COPD lung tissue. These data suggest that CS regulates ciliary proteins in human epithelial cells. To the best of our knowledge, this is the first time that CS has been shown to regulate AurA, HDAC6, and phospho-GSK3 $\beta$ in motile cilia or in human airway epithelial cells.

HDAC6 has been shown to regulate primary cilia resorption in response to extracellular stress (69) as well as the autophagic pathway through autophagosome-lysosome fusion (38). We therefore 
chose to further evaluate the role of HDAC6 on cilia length in our in vitro and in vivo models. Hdac $\sigma^{-/ Y}$ MTECs and $H$ dac $^{-/ Y}$ mice, which display an autophagy-deficient phenotype, were protected against CS-induced cilia shortening. These results are consistent with a role for autophagy in CS-induced cilia shortening.

HDAC6 has dual functionality as a ubiquitin-binding protein and as a regulator of protein acetylation (70). The ubiquitin-binding activity of HDAC6 is critical for its function in the transport of ubiquitinated proteins to aggresomes, thereby facilitating cellular adaptive responses to the accumulation of misfolded proteins (70). Consistently, we observed that the binding of HDAC6 to ubiquitinated proteins increases upon CS exposure. In agreement with prior observations (36), the organization of aggregated protein into aggresomal structures was impaired in $H_{d a c 6^{-}} Y$ cells, as evidenced by the loss of perinuclear aggresome puncta and increased protein ubiquitination. We furthermore found that HDAC6 expression was upregulated in a genetic model of oxidative stress susceptibility in $\mathrm{Nrf2^{-/- }}$ mice. HDAC6 expression was consistently elevated in $\mathrm{Nrf2}^{-{ }^{--}}$mice tissues and cells, which also displayed increased protein ubiquitination. Interestingly, NRF2 inhibition increases autophagy (71), and increasing NRF2 represses CSEinduced autophagosome formation (72). These results suggest that HDAC6 expression is increased by oxidative and proteostatic stress and may be counterregulated by NRF2.

We hypothesized that the ubiquitin-binding function of HDAC6 may override its deacetylase activity during CS-induced cellular stress. Despite an increase in HDAC6 protein expression, CS did not reduce the amount of acetylated $\alpha$-tubulin, a known HDAC6 substrate, in HBE cells or MTECs. To determine how HDAC6 deacetylase activity could be impaired in our model, we investigated whether HDAC6 is posttranslationally modified in response to CS. Using our in vivo CS exposure model, we demonstrated that HDAC6 is acetylated in response to CS, a modification that has been shown to inhibit its tubulin deacetylase activity and retain HDAC6 in the cytoplasm (73). Here, we implicate sirtuin 1 (SIRT1), an $\mathrm{NAD}^{+}$-dependent protein/histone deacetylase, in the regulation of the acetylation state of HDAC6. SIRT1 expression has been shown to be decreased in lungs of patients with COPD $(54,55)$. Our data implicate SIRT1 as an HDAC6 deacetylase and suggest a model in which HDAC6 deacetylase activity is reduced by $\mathrm{CS}$ as a result of SIRT1 downregulation, resulting in acetylation and catalytic inhibition of HDAC6 (74). Interestingly, SIRT1 inhibition has been shown to enhance autophagy induced by CS in the lungs of Sirt $1^{+/-}$mice (75). Taken together, these data suggest that CS reduces SIRT1, leading to increased acetylation of HDAC6 and increased autophagy.

Our data lend support to a model whereby delivery of cellular proteins by HDAC6 for their processing by autophagy may represent a major mechanism by which CS disrupts the function of the airway epithelium. The autophagic pathway is known to act as a compensatory mechanism for protein degradation under conditions of impaired proteasomal activity, which can be induced by CS $(36,76)$. The accumulation of CS-denatured proteins in aggresomes in excess of cellular degradative capacity may represent a harmful intermediate state. While we show that activation of the proposed ciliophagic pathway leads to cilia shortening, excessive activation of this pathway may ultimately lead to ciliated cell loss and ciliated cell death. Observations from our MTEC model suggest that activation of the autophagic pathway during CS exposure regulates CS-induced cilia shortening at low-dose exposure to CS, but aggravates injury to ciliated epithelial cells at higher-

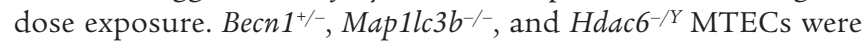
resistant to CS-induced toxicity and apoptosis at high doses of CS. The autophagy inhibitor 3-MA prevented CS-induced cell death at high doses of CS $\left(100 \mathrm{mg} / \mathrm{m}^{3}\right)$, suggesting that autophagy may contribute to ciliated cell death under these conditions. These findings are consistent with our previous observations that epithelial cells from LC3B knockout (Map1lc36 $b^{-/}$) mice were protected from CS-induced apoptosis and that Map1lc $3 b^{-/-}$mice were resistant to CS-induced emphysema development $(10,11)$.

Ciliated airway epithelial cells, which provide MCC function, are of crucial importance in airway physiology and pathophysiology (25). MCC acts as a lung defense mechanism against inhaled particles and pathogens. Methodology to determine MCC in humans may represent an important clinical tool to assess airway function in diseases such as COPD or cystic fibrosis (77). In order to test our hypotheses that autophagy plays a maladaptive role in cilia homeostasis, we implemented a physiological model of airway MCC. We observed that genetic deletion of the autophagic mediators beclin 1 and $\mathrm{LC} 3 \mathrm{~B}$ protected mice from MCC disruption in an in vivo model of CS exposure. Furthermore, genetic or chemical inhibition of HDAC6 also protected mice from the MCC disruption associated with $\mathrm{CS}$ exposure. These results suggest that pharmacological inhibition of HDAC6 may reverse or inhibit CS-induced airway dysfunction. Consistent with our data, HDAC6 inhibitors have been successfully used in some mouse models of neurodegeneration, in which HDAC6 is upregulated to facilitate the aggregation of mutant or misfolded proteins (78).

We demonstrate that the alleviation of CS-induced proteotoxicity prevents CS-induced pathological changes in airway function. Treatment of mice with the chemical chaperone 4-PBA improved MCC following CS exposure. Our data highlight the benefits of using chemical chaperones as therapeutics to preserve the function of critical proteins in response to noxious cellular stresses such as CS (79-81). The preservation of proteins required for cilia formation and/or ciliated cell integrity may reduce autophagic substrates and enhance MCC in smokers. Deletion of the antioxidant response (e.g., Nrf2 $2^{-/-}$mice) reduced MCC function and exacerbated responses to CS. MCC impairment caused by CS exposure was not associated with increased apoptosis in the lung, as indicated by TUNEL analysis. Furthermore, changes in MCC were reversible by smoking cessation in the acute exposure model, indicating that epithelial cell death is not the primary mechanism. The preservation of proteins required for cilia formation and/or ciliated cell function may reduce autophagic substrates and enhance MCC independently of cell death in smokers. We speculate that the maintenance of MCC in response to CS insult may be a critical line of defense in the development of CS-induced bronchitis and in the pathogenesis of COPD.

Previously, we have shown that autophagy may be regulated in human COPD. Specifically, we found that autophagic proteins were upregulated in mild and severe COPD and coincided with the expression of inflammatory or apoptotic markers in severe COPD $(10,82)$. Additionally, we found evidence for increased autophagosome formation in human COPD lung tissues. In the current study, we identify HDAC6, an autophagy mediator, as a novel, highly regulated gene target in COPD. We found that HDAC6 protein was upregulated in human COPD by immunofluorescence analysis of ciliated airway sections and by Western immunoblotting of total lung homogenates. Fur- 
thermore, at the epigenetic level, we found that HDAC6 was hypomethylated in human COPD patients (from DNA methylation array data) compared with controls. Hypomethylation of HDAC6 may represent a smoke-related phenomenon present in patients with COPD and may be sex dependent.

Finally, in this study, we show that COPD severity is associated with a general increase in protein aggregates and inclusion bodies in human COPD lung tissues. These data are consistent with previous findings that demonstrate a substantial increase in ubiquitinated proteins in severely emphysematous human lung tissue sections $(9,12)$. Similar dysfunctional proteostatic mechanisms have also been implicated in the pathogenesis of CS-induced chronic bronchitis, which involves the aggresomal sequestration of cystic fibrosis transmembrane conductance regulator (CFTR) $(76,83,84)$ and in other chronic lung diseases such as $\alpha 1$-antitrypsin deficiency (A1AD) and pulmonary fibrosis (5). We conclude that certain features of our in vitro and in vivo findings were corroborated in human clinical samples, namely that patients with COPD displayed increased HDAC6 expression and evidence for proteopathy in addition to elevated autophagy, as previously described $(10,11)$.

We conclude that ciliophagy, an HDAC6-dependent autophagic pathway, represents what we believe to be a novel pathway that is critical to cilia homeostasis in response to CS exposure and suggests a potential therapeutic target for airway disease caused by CS exposure.

\section{Methods}

Animals. Becn1 $1^{+/}$mice were from Beth Levine (University of Texas Southwestern Medical Center at Dallas, Dallas, Texas, USA). The $H_{d a c 6^{-}-Y}$ mice, bearing an $\mathrm{X}$ chromosome deletion of HDAC6, were obtained from Bin Shan (Tulane University, New Orleans, Louisiana, USA). Map $1 l c 3 b^{-/-}$mice were from Marlene Rabinovitch (Stanford University, Stanford, California, USA) and were backcrossed to C57BL/6 mice. The $\mathrm{Nrf2^{-/- }}$ mice were from Shyam Biswal (Johns Hopkins University Medical Center, Baltimore, Maryland, USA). The GFP-LC3B mice were from RIKEN BioResource Center. WT C57BL/6 mice were obtained from The Jackson Laboratory.

In vivo CS exposure and chemical treatments. Age- and sex-matched mice (6-12 weeks of age) were exposed to RA or CS in whole-body exposure chambers as described for 2 hours per day $\left(150 \mathrm{mg} / \mathrm{m}^{3}\right), 5$ days per week, for 1 week, 3 weeks, 2 months, and 6 months $(10,11)$. 4-PBA (1\% w/v) solution was administered by replacing the animal's water for the duration of CS exposure. Animals were checked daily and the quantity of water consumed compared with that of the control RA mice. Water solutions were changed twice weekly. Tubastatin A $(25 \mathrm{mg} / \mathrm{kg})$ was administered by daily i.p. injections prior to CS exposure. At the end of each exposure regimen, the left lung was isolated, and the right lungs were inflated, dissected, and fixed in $4 \%$ formalin. The proximal trachea up to the larynx and a portion of the proximal part of the left lung were fixed for TEM.

Cell culture and CSE treatment. Human BEAS-2B lung epithelial cells and mouse lung fibroblasts were maintained in DMEM supplemented with $10 \% \mathrm{FBS}$ and gentamicin $(50 \mathrm{~g} / \mathrm{ml})$. CSE was prepared and added to culture media as previously described $(10,11)$. Cigarette smoke condensate (CSC) was prepared and added to culture media as previously described (8).

Generation and CS exposure of MTEC cultures. The methods for MTEC isolation and culture generation have been previously described $(39,85)$. MTECs were proliferated and differentiated at an ALI in Transwell cultures (see Supplemental Methods for details). Exposure of MTEC ALI cultures was conducted in a custom-designed humidified chamber (EMI Services) at $37^{\circ} \mathrm{C}$ by exposing the cells for 10 minutes to 50 or $100 \mathrm{mg} / \mathrm{m}^{3}$ of mainstream CS from 3R4F research-reference filtered cigarettes.
Immunoprecipitation and immunoblot analyses. Immunoprecipitation and immunoblot analyses were performed as previously described, with minor modifications (10). Detailed methods for the isolation of protein extracts, lysosome-enriched (LE) fractions, immunoprecipitation, and immunoblotting have been previously described (43) and are provided in the Supplemental Methods (43).

In vivo autophagic flux assay. Methods for in vivo autophagic flux assays have been described previously (44). Briefly, mice exposed to CS or RA were given an i.p. injection of $40 \mathrm{mg} / \mathrm{kg}$ leupeptin in saline 1 hour or 24 hours following the final CS treatment. Control mice received an equal volume of the vehicle. Lung tissues from the leupeptin-treated RA and CS mice were harvested 2 hours later. The tissues were flash-frozen, and LC3B turnover was assessed in the LE fraction. To quantify the LC3B levels, we performed Western blot analysis, with a standard curve consisting of purified GSTLC3B protein. Autophagic flux was defined as the difference in LC3B-II quantity on Western blots obtained in the presence and absence of an inhibitor (see Supplemental Methods for details).

In vitro autophagic flux assay. Following exposure of MTEC cultures to CS $\left(50 \mathrm{mg} / \mathrm{m}^{3}\right)$ or BEAS-2B cells to $20 \%$ CSE, cells were treated for 10 minutes with CQ $(25 \mu \mathrm{M})$ before harvesting. Control cells were treated with CQ in the absence of CS treatment. The samples were then harvested in RIPA buffer from control and treated cells at the same time: 0 and 1 hour after treatment for MTECs; and 0, 1, 4, or 8 hours after treatment for BEAS-2B cells. Lysates were then analyzed by standard immunoblot assays for LC3B expression. Autophagic flux was defined as the difference in LC3B-II quantity (densitometer units) on Western blots obtained in the presence and absence of an inhibitor.

Isolation of autophagosomes from LC3B-GFP mice. GFP-LC3B autophagosomes were isolated using a modified version of a previously described method (ref. 86 and see Supplemental Methods). Briefly, autophagosome/lysosome-enriched fractions were generated from the lungs of LC3B-GFP mice exposed to RA or CS for 3 weeks. LC3B-positive autophagosomes/lysosomes were immunoprecipitated using GFP-conjugated $\mu \mathrm{MACS}$ microbeads (Miltenyi Biotec), isolated using the magnetic field of a $\mu$ MACS separator, and analyzed by SDS electrophoresis followed by Western immunoblot analysis.

Cell viability and cytotoxicity assays. Cytotoxicity was assessed by measuring LDH activity in the basal media of the MTEC cultures (Cytotoxicity Detection Kit ${ }^{\text {PLus; }}$ Roche Diagnostics) according to the manufacturer's protocol. Apoptosis was detected using the Annexin V FITC Apoptosis Detection Kit (BioVision) according to the manufacturer's instructions. The proportion of viable and apoptotic cells was assessed using a FACS Canto II (BD Biosciences) and FlowJo analytical software (TreeStar Inc.).

Immunofluorescence staining. Cells were fixed and analyzed by immunofluorescence microscopy using standard methods (10, 11). Misfolded protein aggregates were stained using a recently developed dye and the ProteoStat Aggresome Detection Kit for flow cytometry and microscopy (Enzo Life Sciences). Slides were imaged using epifluorescence (DM LB microscope and DFC 480 3CCD Color Vision Module; Leica) or a confocal microscope (Zeiss LSM 510 with 2-photon; Carl Zeiss). Puncta quantification per field was determined using an ImageJ macro (NIH) described in detail in the Supplemental Methods.

Immunogold and TEM. TEM samples were fixed with $2 \%$ formaldehyde and $2.5 \%$ glutaraldehyde in $0.1 \mathrm{M}$ sodium cacodylate buffer, $\mathrm{pH}$ 7.4. Immunogold TEM cells were fixed with 4\% PFA in sodium phosphate ( $\mathrm{pH} 7.4)$ and labeled with IFT88 antibody 1:20 prior to embedding. A detailed embedding protocol is provided in the Supplemental Methods. The samples were imaged using a Technai G2 Spirit BioTWIN TEM. For EM quantification, 10-15 image fields were selected for each mouse or sample. Autophagosomes were quantified from ciliated cells. The total area of the 
image was calculated, and the amount of autophagosomes per unit area were counted using ImageJ software.

SEM. MTEC cultures were fixed and mounted for SEM as described in the Supplemental Methods. Specimens were imaged with a JEOL 9355 Field Emission Zeiss Gun SEM.

Immunobistochemistry staining. Formalin-fixed, paraffin-embedded 5-micron-thick lung sections were prepared and incubated with goat anti-HDAC6 (Santa Cruz Biotechnology Inc.) primary antibodies as described in the Supplemental Methods. The threshold feature in ImageJ was used to measure differences in HDAC6-staining intensity.

Measurement of cilia lengths in vitro and in vivo. The average length of cilia in the SEM images ( $\times 1000$ magnification) of MTEC cultures exposed to RA or CS in vitro was calculated by measuring a minimum of 3 cilia on each ciliated cell in each image using ImageJ software tools $(n=5$ images per sample). The distribution of cilia length in MTECs was determined using the frequency histogram distribution tool in GraphPad Prism (GraphPad Software) by parsing into bins of $0.2 \mathrm{AU}$. The average length of cilia in the control MTECs and in the MTECs exposed to $50 \mathrm{mg} / \mathrm{m}^{3}$ or $100 \mathrm{mg} / \mathrm{m}^{3} \mathrm{CS}$ was calculated by combining the average cilia length per cell (3-10 ciliated cells per image) in each image ( $n=5$ images per sample) and normalizing to control values. To accurately assess the extent of cilia shortening in the MTECs, we used an empiric threshold of $0.6 \mathrm{AU}$ (chosen as the upper bound of the lower tail of a Gaussian fit to the length distribution) to filter the cilia length distribution into "short" and "long" and excluded the "short" cilia to maximize the signal-to-noise ratio for the change in cilia length $(25,40)$. Using this cutoff, only cilia with a length between 0.6 and $3 \mathrm{AU}$ (cells with "long" cilia) were counted and normalized to control values.

The average length of cilia in H\&E-stained airways ( $\times 60$ magnification) of mice exposed to RA or CS in vivo was calculated by measuring a minimum of 3 cilia on each ciliated cell in each image using ImageJ tools ( $n=5$ images per mouse). Similarly, the distribution of cilia length in vivo was determined using the frequency histogram distribution tool in GraphPad Prism by parsing into bins of $0.4 \mathrm{AU}$. The average length of cilia in the airways of mice exposed to RA or in the airways of mice exposed to CS was calculated by combining the average cilia length per cell (3-10 ciliated cells per image) in each image ( $n=5$ images per mouse) and normalizing to RA control values.

Assessment of MCC in vivo. MCC was quantified using a noninvasive oropharyngeal aspiration procedure described previously $(56,87,88)$, with minor modifications as described in the Supplemental Methods. Briefly, mice were anesthetized, and $50 \mu \mathrm{l}$ of normal saline containing approximately $0.3-0.5 \mathrm{mCi}$ of ${ }^{99 \mathrm{~m}} \mathrm{Tc}$-sc was introduced into the distal part of the oropharynx and aspirated. Mouse lungs were imaged immediately after aspiration ( $t=0$ hour) and at 1 and 3 hours. Whole-mouse 3D $\mu$ SPECT images from the 0,1 -, and 3 -hour time points were obtained, reconstructed, analyzed, and expressed as the percentage removed by MCC, as described in the Supplemental Methods. Dual-probe SPECT imaging was performed to obtain anatomic registration of the instilled lungs with respect to the thoracic skeleton using ${ }^{99 \mathrm{~m}} \mathrm{Tc}$-mdp, a standard SPECT skel-

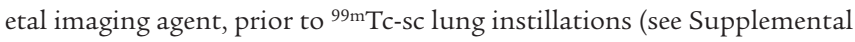
Methods for a detailed description).

CHARM. CHARM methylation array data from 247 lung samples (containing 124 COPD and 43 control samples) were loaded into $\mathrm{R}$ using the CHARM Bioconductor package. CHARM quality control, normalization, and smoothing (60) were used to calculate the percentage of methylation at each of the 2,162,405 probe sites distributed across the genome. Only data from COPD and control samples were used for the differential methylation analysis. At each probe, we logit-transformed the percentage of methylation and fitted a linear model using the methylation level as outcome and the COPD case-control status as the predictor, adjusting for age, sex, and pack-years of smoking.
The $P$ values of adjacent probes were combined into differentially methylated regions (DMRs) using the Comb-p python package (61) (GitHub; https://github.com/brentp/combined-pvalues/). Comb-p uses Kechris's method (89), a modification of the Stouffer-Liptak method for combining $P$ values, in which weights are assigned using the observed autocorrelation in sliding windows across the genome. The DMRs were used to create a custom track in the UCSC Genome Browser; DMRs with at least 50\% overlap with conserved human-mouse-rat transcription factor binding sites (TFBS-conserved track) were selected (22 DMRs total). Each DMR was associated with the nearest gene (within $10 \mathrm{~kb}$ ).

Statistical analysis. Statistical analysis was conducted using GraphPad Prism. Data are presented as the mean \pm SEM from at least three independent experiments. Differences in measured variables between experimental and control groups were assessed using the Student's $t$ test and between multiple groups and conditions using one-way and two-way ANOVA and Bonferroni's post tests. For MCC assays, a significant $P$ value was followed by a pairwise comparison using a two-sample $t$ test for a priori hypotheses only. Thus, no adjustment in the significance level was made for multiple comparisons. $P$ values were calculated, and minimum statistical significance was accepted at $P<0.05$.

Patients. Patient samples were classified based on the GOLD guidelines (90) and were obtained from the Lung Tissue Research Consortium (LTRC) (Supplemental Table 1).

Study approval. All animal experimental protocols were approved by the Harvard Standing Committee for Animal Welfare. Human lung tissue experiments were approved by the IRB of Brigham and Women's Hospital.

\section{Acknowledgments}

Confocal imaging and analysis were performed at the Harvard NeuroDiscovery Center. We thank Bradley Yoder for providing the IFT88 antibody and Donna Beer Stolz for her technical help with imaging analysis. Marlene Rabinovich and Beth Levine provided the Map1lc3b $b^{-/-}$and Becn1 $1^{+-}$mice, respectively. We thank Emeka Ifedigbo, Stephen C. Moore, Hari Parameswaran, and Shivraj Tyagi for providing significant technical support. We also thank Bonna Ith and Timelia Tison for slide preparation and staining and Ludo Van Den Bosch, Constantin van Outryve d'Ydewalle, Richard Austin, and Sana Basseri for their advice on in vivo therapy regimes. S.W. Ryter received salary support from the Lovelace Respiratory Research Institute/Brigham and Women's Hospital (LRRI/BWH) consortium for lung research. This work was supported by NIH grants R01-HL60234, R01-HL55330, R01-HL079904, and P01-HL70807, as well as by a FAMRI clinical innovator award (to A.M.K. Choi). H.C. Lam was supported by an NIH T32 training grant awarded to the Harvard School of Public Health (HL007118), an AHA predoctoral grant (09PRE2250120), and by the Lovelace Respiratory Research Institute. J. Quackenbush and E.K. Silverman were supported by NIH grant P01 HL105339 (to E.K. Silverman).

Received for publication March 1, 2013, and accepted in revised form August 30, 2013.

Address correspondence to: Augustine M.K. Choi, Department of Medicine, Weill Cornell Medical College, 525 East 68th Street, Room M-522, Box 130, New York, New York 10065, USA. Phone: 212.746.4720; Fax: 212.746.8793; E-mail: amc2056@med.cornell.edu.

Hong Pyo Kim's present address is: Hong Pyo Kim, School of Pharmacy, Ajou University, Republic of Korea. 
1. Dal-Re R. Worldwide behavioral research on major global causes of mortality. Health Edu Behav. 2001; 38(5):433-440.

2. Barnes PJ, Shapiro SD, Pauwels RA. Chronic obstructive pulmonary disease: molecular and cellular mechanisms. Eur Resp J. 2003;22(4):672-688.

3 . Barnes PJ. Chronic obstructive pulmonary disease NEngl J Med. 2000;343(4):269-280.

4. Hurst JR, et al. Susceptibility to exacerbation in chronic obstructive pulmonary disease. New EnglJ Med. 2010;363(12):1128-1138.

5. Bodas M, Tran I, Vij N. Therapeutic strategies to correct proteostasis-imbalance in chronic obstructive lung diseases. Curr Mol Med. 2012;12(7):807-814.

6. Min T, Bodas M, Mazur S, Vij N. Critical role of proteostasis-imbalance in pathogenesis of COPD and severe emphysema. J Mol Med. 2011;89(6):577-593.

7. van Rijt SH, et al. Acute cigarette smoke exposure impairs proteasome function in the lung. Am J Physiol Lung Cell Mol Physiol. 2012;303(9):L814-L823.

8. Malhotra D, et al. Heightened endoplasmic reticulum stress in the lungs of patients with chronic obstructive pulmonary disease: the role of Nrf2-regulated proteasomal activity. Am J Respir Crit Care Med. 2009;180(12):1196-1207.

9. Somborac-Bacura A, et al. Cigarette smoke induces endoplasmic reticulum stress response and proteasomal dysfunction in human alveolar epithelial cells. Exp Physiol. 2013;98(1):316-325.

10. Chen $\mathrm{ZH}$, et al. Egr-1 regulates autophagy in cigarette smoke-induced chronic obstructive pulmonary disease. PloS One. 3008;3(10):e3316.

11. Chen $\mathrm{ZH}$, et al. Autophagy protein microtubule-associated protein 1 light chain-3B (LC3B) activates extrinsic apoptosis during cigarette smoke-induced emphysema. Proc Natl Acad Sci U S A. 2010; 107(44):18880-18885.

12. Fujii $\mathrm{S}$, et al. Insufficient autophagy promotes bronchial epithelial cell senescence in chronic obstructive pulmonary disease. Oncoimmunol. 2012; 1(5):630-641.

13. Monick MM, et al. Identification of an autophagy defect in smokers' alveolar macrophages. JImmunol. 2010;185(9):5425-5435

14. Mizushima N, Komatsu M. Autophagy: renovation of cells and tissues. Cell. 2011;147(4):728-741.

15. Yang Z, Levine B, Kroemer G. Autophagy in the pathogenesis of disease. Cell. 2008;132(1):27-42.

16. Klionsky DJ. Mammalian autophagy: core molecular machinery and signaling regulation. Curr Opin Cell Biol. 2010;22(2):124-131.

17. Liang XH., Jackson S, Seaman M, Brown K, Kempkes B, Hibshoosh H, Levine B. Induction of autophagy and inhibition of tumorigenesis by beclin 1. Nature. 1999;402(6762):672-676.

18. He C, Levine B. The Beclin 1 interactome. Curr Opin Cell Biol. 2010;22(2):140-149.

19. Kabeya Y, Mizushima N, Yamamoto A, Oshitani-Okamoto S, Ohsumi Y, Yoshimori T. LC3, GABARAP and GATE16 localize to autophagosomal membrane depending on form-II formation. J Cell Sci. 2004;117(pt 13):2805-2812.

20. Clague MJ, Urbe S. Ubiquitin: same molecule, different degradation pathways. Cell. 2010; 143(5):682-685.

21. Kraft C, Peter M, Hofmann K. Selective autophagy: ubiquitin-mediated recognition and beyond. Nature Cell Biol. 2010;12(9):836-841.

22. Kim PK, Hailey DW, Mullen RT, Lippincott-Schwartz J. Ubiquitin signals autophagic degradation of cytosolic proteins and peroxisomes. Proc Natl Acad Sci U S A. 2008;105(52):20567-20574.

23. Pankiv S, et al. p62/SQSTM1 binds directly to Atg8/LC3 to facilitate degradation of ubiquitinated protein aggregates by autophagy. J Biol Chem. 2007; 282(33):24131-24145.

24. Lamark T, Johansen T. Aggrephagy: selective disposal of protein aggregates by macroautophagy. Int
J Cell Biol. 2012;2012:736905.

25. Leopold PL, O'Mahony MJ, Lian XJ, Tilley AE, Harvey BG, Crystal RG. Smoking is associated with shortened airway cilia. PloS One. 2009;4(12):e8157.

26. Frasca JM, Auerbach O, Carter HW, Parks VR. Morphologic alterations induced by short-term cigarette smoking. Am J Pathol. 1983;111(1):11-20.

27. Ballenger JJ. Experimental effect of cigarette smoke on human respiratory cilia. New Engl J Med. 1960; 263:832-835.

28. Haswell E, Hewitt K, Thorne D, Richter A, Gaca MD. Cigarette smoke total particulate matter increases mucous secreting cell numbers in vitro: a potential model of goblet cell hyperplasia. Toxicol In Vitro. 2010;24(3):981-987.

29. Martinez FJ, Donohue JF, Rennard SI. The future of chronic obstructive pulmonary disease treatment - difficulties of and barriers to drug development. Lancet. 2011;378(9795):1027-1037.

30. Ishikawa H, Marshall WF. Ciliogenesis: building the cell's antenna. Nat Rev Mol Cell Biol. 2011; 12(4):222-234.

31. Scholey JM, Anderson KV. Intraflagellar transport and cilium-based signaling. Cell. 2006; 125(3):439-442.

32. Huang K, Diener DR, Rosenbaum JL. The ubiquitin conjugation system is involved in the disassembly of cilia and flagella. J Cell Biol. 2009;186(4):601-613.

33. Pugacheva EN, Jablonski SA, Hartman TR, Henske EP, Golemis EA. HEF1-dependent Aurora A activation induces disassembly of the primary cilium. Cell. 2007;129(7):1351-1363.

34. Thoma CR, Frew IJ, Hoerner CR, Montani M, Moch $\mathrm{H}$, Krek W. pVHL and GSK3 $\beta$ are components of a primary cilium-maintenance signalling network. Nat Cell Biol. 2007;9(5):588-595.

35. Trüe O, Matthias P. Interplay between histone deacetylases and autophagy-from cancer therapy to neurodegeneration. Immunol Cell Biol. 2012; 90(1):78-84

36. Kawaguchi Y, Kovacs JJ, McLaurin A, Vance JM, Ito A, Yao TP. The deacetylase HDAC6 regulates aggresome formation and cell viability in response to misfolded protein stress. Cell. 2003; 115(6):727-738

37. Pandey UB, et al. HDAC6 rescues neurodegeneration and provides an essential link between autophagy and the UPS. Nature. 2007; 447(7146):859-863.

38. Lee JY, et al. HDAC6 controls autophagosome maturation essential for ubiquitin-selective qualitycontrol autophagy. EMBO J. 2010;29(5):969-980.

39. Lam HC, Choi AM, Ryter SW. Isolation of mouse respiratory epithelial cells and exposure to experimental cigarette smoke at air liquid interface. J Vis Exp. 2011;48(48):2513.

40. Satir P. Studies on cilia. 3. Further studies on the cilium tip and a "sliding filament" model of ciliary motility. J Cell Biol. 1968;39(1):77-94.

41. Klionsky DJ, et al. Guidelines for the use and interpretation of assays for monitoring autophagy. Autophagy. 2012;8(4):445-544.

42. Klionsky DJ, Cuervo AM, Seglen PO. Methods for monitoring autophagy from yeast to human. Autophagy. 2007;3(3):181-206.

43. Perry CN, Kyoi S, Hariharan N, Takagi H, Sadoshima J, Gottlieb RA. Novel methods for measuring cardiac autophagy in vivo. Meth Enzymol. 2009; 453:325-342.

44. Haspel J, et al. Characterization of macroautophagic flux in vivo using a leupeptin-based assay. Autophagy. 2011;7(6):629-642.

45. Qu X, et al. Promotion of tumorigenesis by heterozygous disruption of the beclin 1 autophagy gene. J Clin Invest. 2003;112(12):1809-1820.

46. Kim S, Tsiokas L. Cilia and cell cycle re-entry: more than a coincidence. Cell Cycle. 2011; 10(16):2683-2690.
47. Kim HP, et al. Autophagic proteins regulate cigarette smoke-induced apoptosis: protective role of heme oxygenase-1. Autophagy. 2008;4(7):887-895.

48. Ouyang $\mathrm{H}$, et al. Protein aggregates are recruited to aggresome by histone deacetylase 6 via unanchored ubiquitin C termini. J Biol Chem. 2012; 287(4):2317-2327.

49. Boyault $C$, et al. HDAC6 controls major cell response pathways to cytotoxic accumulation of protein aggregates. Genes Dev. 2007;21(17):2172-2181.

50. Johnston JA, Ward CL, Kopito RR. Aggresomes: a cellular response to misfolded proteins. J Cell Biol. 1998;143(7):1883-1898.

51. Rangasamy T, et al. Genetic ablation of Nrf2 enhances susceptibility to cigarette smoke-induced emphysema in mice. J Clin Invest. 2004; 114(9):1248-1259.

52. Suzuki $M$, et al. Down-regulated NF-E2-related factor 2 in pulmonary macrophages of aged smokers and patients with chronic obstructive pulmonary disease. Am J Respir Cell Mol Biol. 2008; 39(6):673-682.

53. Goven $D$, et al. Altered Nrf2/Keap1-Bach1 equilibrium in pulmonary emphysema. Thorax. 2008; 63(10):916-924.

54. Yao $\mathrm{H}$, et al. SIRT1 protects against emphysema via FOXO3-mediated reduction of premature senescence in mice. J Clin Invest. 2012;122(6):2032-2045.

55. Kawai Y, Garduno L, Theodore M, Yang J, Arinze IJ. Acetylation-deacetylation of the transcription factor $\mathrm{Nrf2}$ (nuclear factor erythroid 2-related factor 2) regulates its transcriptional activity and nucleocytoplasmic localization. J Biol Chem. 2011; 286(9):7629-7640.

56. Foster WM, Walters DM, Longphre M, Macri K, Miller LM. Methodology for the measurement of mucociliary function in the mouse by scintigraphy. J Appl Physiol. 2001;90(3):1111-1117.

57. Wiley JC, Pettan-Brewer C, Ladiges WC. Phenylbutyric acid reduces amyloid plaques and rescues $\operatorname{cog}$ nitive behavior in AD transgenic mice. Aging Cell. 2011;10(3):418-428.

58. Ozcan U, et al. Chemical chaperones reduce ER stress and restore glucose homeostasis in a mouse model of type 2 diabetes. Science. 2006; 313(5790):1137-1140.

59. Irizarry RA, et al. Comprehensive high-throughput arrays for relative methylation (CHARM). Genome Res. 2008;18(5):780-790.

60. Aryee MJ, et al. Accurate genome-scale percentage DNA methylation estimates from microarray data. Biostatistics. 2011;12(2):197-210.

61. Pedersen BS, Schwartz DA, Yang IV, Kechris KJ. Comb-p: software for combining, analyzing, grouping and correcting spatially correlated P-values. Bioinformatics. 2012;28(22):2986-2988.

62. Counts JL, Goodman JI. Hypomethylation of DNA: a possible epigenetic mechanism involved in tumor promotion. Prog Clin Biol Res.1995;391:81-101.

63. Choi AM, Ryter SW, Levine B. Autophagy in human health and disease. $N$ Engl J Med. 2013; 368(7):651-662.

64. Sisson JH, et al. Smoke and viral infection cause cilia loss detectable by bronchoalveolar lavage cytology and dynein ELISA. Am J Respir Crit Care Med. 1994;149(1):205-213.

65. Abdi S, Evans MJ, Cox RA, Lubbesmeyer H, Herndon DN, Traber DL. Inhalation injury to tracheal epithelium in an ovine model of cotton smoke exposure. Early phase (30 minutes). Am Rev Respir Dis. 1990;142(6 pt 1):1436-1439.

66. Inoue D, et al. Inducible disruption of autophagy in the lung causes airway hyper-responsiveness. Biochem Biophys Res Commun. 2011;405(1):13-18.

67. DiBella LM, Park A., Sun Z. Zebrafish Tsc1 reveals functional interactions between the cilium and the TOR pathway. Human Mol Genet. 2009; 18(4):595-560. 
68. Yuan S, Li J, Diener DR, Choma MA, Rosenbaum JL, Sun Z. Target-of-rapamycin complex 1 (Torc1) signaling modulates cilia size and function through protein synthesis regulation. Proc Natl Acad Sci U S A. 2012;109(6):2021-2026.

69. Prodromou NV, et al. Heat shock induces rapid resorption of primary cilia. J Cell Sci. 2012; 125(pt 18):4297-4305.

70. Li G, Jiang H, Chang M, Xie H, Hu L. HDAC6 $\alpha$-tubulin deacetylase: a potential therapeutic target in neurodegenerative diseases. J Neurol Sci. 2011; 304(1-2):1-8.

71. Rao VA, et al. The antioxidant transcription factor nrf2 negatively regulates autophagy and growth arrest induced by the anticancer redox agent mitoquinone. J Biol Chem. 2010; 285(45):34447-34459.

72. Zhu L, Barret EC, Xu Y, Liu Z, Manoharan A, Chen Y. Regulation of cigarette smoke (cs)-induced autophagy by nrf2. PLoS One. 2013;8(4):e55695.

73. Liu Y, Peng L, Seto E, Huang S, Qiu Y. Modulation of histone deacetylase 6 (HDAC6) nuclear import and tubulin deacetylase activity through acetylation. J Biol Chem. 2012;287(34):29168-29174.

74. Gal J, Chen J, Barnett KR, Yang L, Brumley E, Zhu H. HDAC6 regulates mutant SOD1 aggregation through two SMIR motifs and tubulin acetylation. J Biol Chem. 2013;288(21):15035-15045.

75. Hwang JW, et al. Cigarette smoke-induced autophagy is regulated by SIRT1-PARP-1-dependent mechanism: implication in pathogenesis of COPD. Arch Biochem Biophys. 2010;500(2):203-209.

76. Liu XD, et al. Transient aggregation of ubiquitinated proteins is a cytosolic unfolded protein response to inflammation and endoplasmic reticulum stress. J Biol Chem. 2012;287(23):19687-19698.

77. Donaldson SH, Corcoran TE, Laube BL, Bennett WD. Mucociliary clearance as an outcome measure for cystic fibrosis clinical research. Proc Am Thorac Soc. 2007;4(4):399-405.

78. d'Ydewalle C, Bogaert E, Van Den Bosch L. HDAC6 at the intersection of neuroprotection and neurodegeneration. Traffic. 2012;13(6):771-779.

79. Stanojkovic I, et al. Pulmonary function, oxidative stress and inflammatory markers in severe COPD exacerbation. Respir Med. 2011;105(suppl 1):S31-S37.

80. Cohen FE, Kelly JW. Therapeutic approaches to protein-misfolding diseases. Nature. 2003; 426(6968):905-909.

81. Mu TW, et al. Chemical and biological approaches synergize to ameliorate protein-folding diseases. Cell. 2008;134(5):769-781.

82. An $\mathrm{CH}$, et al. TLR4deficiency promotes autophagy during cigarette smoke-induced pulmonary emphysema. Am J Physiol Lung Cell Mol Physiol. 2012; 303(9):L748-L757

83. Bartoszewski R, Rab A, Fu L, Bartoszewska S, Collawn J, Bebok Z. CFTR expression regulation by the unfolded protein response. Meth Enzymol. 2011; 491:3-24.
84. Clunes LA, et al. Cigarette smoke exposure induces CFTR internalization and insolubility, leading to airway surface liquid dehydration. FASEB J. 2012; 26(2):533-545.

85. You Y, Richer EJ, Huang T, Brody SL. Growth and differentiation of mouse tracheal epithelial cells: selection of a proliferative population. Am J Physiol: Lung Cell Mol Physiol. 2002;283(6):L1315-L1321.

86. Dengjel J, et al. Identification of autophagosome-associated proteins and regulators by quantitative proteomic analysis and genetic screens. Mol Cell Proteomics. 2012;11(3):M111.014035.

87. Bhashyam AR, et al. Vagal control of mucociliary clearance in murine lungs: a study in a chronic preparation. Auton Neurosci. 2010;154(1-2):74-78.

88. Bhashyam AR, et al. A pilot study to examine the effect of chronic treatment with immunosuppressive drugs on mucociliary clearance in a vagotomized murine model. PloS One. 2012;7(9):e45312

89. Kechris KJ, Biehs B, Kornberg TB. Generalizing moving averages for tiling arrays using combined p-value statistics. Stat Appl Genet Mol Biol. 2010; 9:Article29.

90. Pauwels RA, Buist AS, Calverley PM, Jenkins CR, Hurd SS. Global strategy for the diagnosis, management, and prevention of chronic obstructive pulmonary disease. NHLBI/WHO Global Initiative for Chronic Obstructive Lung Disease (GOLD) Workshop summary. Am J Respir Crit Care Med. 2001; 163(5):1256-1276. 